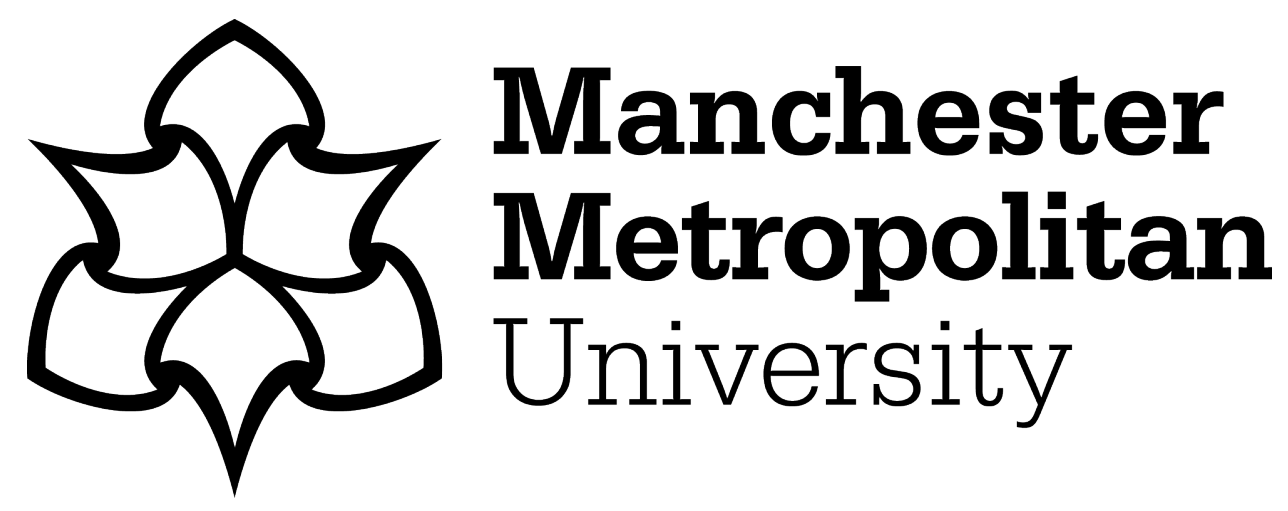

Azhar, M, Dawood, H, Dawood, H, Choudhary, GI, Bashir, AK and Chauhdary, SH (2019) Detail-preserving switching algorithm for the removal of random-valued impulse noise. Journal of Ambient Intelligence and Humanized Computing, 10 (10). pp. 3925-3945. ISSN 1868-5137

Downloaded from: https://e-space.mmu.ac.uk/622928/

Version: Accepted Version

Publisher: Springer (part of Springer Nature)

DOI: https://doi.org/10.1007/s12652-018-1153-0

Please cite the published version 


\title{
Detail-preserving switching algorithm for the removal of random-valued impulse noise
}

\author{
Marium Azhar ${ }^{\mathrm{a}}$, Hassan Dawood ${ }^{\mathrm{a}}$, Hussain Dawood ${ }^{\mathrm{b}}$, Gulraiz Iqbal Choudhary ${ }^{\mathrm{b}}$, Ali \\ Kashif Bashirc, Sajjad Hussain Chauhdary ${ }^{b}$ \\ aDepartment of Software Engineering, University of Engineering and Technology, Taxila 47050, Pakistan. \\ ${ }^{b}$ Faculty of Computing and Information Technology University of Jeddah, Jeddah, Saudi Arabia. \\ ${ }^{\mathrm{c}}$ Department of Science and Technology University of the Faroe Islands, Denmark. \\ *hasandawod@yahoo.com
}

\begin{abstract}
This paper presents a new algorithm for the denoising of images corrupted with random-valued impulse noise (RVIN). It employs a switching approach that identifies the noisy pixels in the first stage and then estimates their intensity values to restore them. Local statistics of the textons in distinct orientations of the sliding window are exploited to identify the corrupted pixels in an iterative manner; using an adaptive threshold range. Textons are formed by using an isometric grid of minimum local distance that preserves the texture and edge pixels of an image, effectively. At the noise filtering stage, fuzzy rules are used to obtain the noise-free pixels from the proposed tridirectional pixels to estimate the intensity values of identified corrupted pixels. The performance of the proposed denoising algorithm is evaluated on a variety of standard gray-scale images under various intensities of RVIN by comparing it with state-of-the-art denoising methods. The proposed denoising algorithm also has robust denoising and restoration power on biomedical images such as, MRI, X-Ray and CT-Scan. The extensive simulation results based on both quantitative measures and visual representations depict the superior performance of the proposed denoising algorithm for various noise intensities.
\end{abstract}

Keywords Random-valued impulse noise, Texton, Tri-directional pixels, Local similarity, Detail-preserving, Fuzzy rules, Medical image.

\section{Introduction}

The field of digital image processing has grown so rapidly and become progressively more important in many other fields including remote sensing, digital telecommunication, medical imaging, graphic printing, security and defense applications. Digital images often get contaminated by impulse noise (IN) during the process of acquisition, encoding, storage and transmission of images(Gonzalez, 2003). Therefore, it is essential to remove such type of noise from images to use them in various other fields. Noise can also contaminate the biomedical images that could be salt-and-pepper noise, speckle noise, Gaussian noise or random-valued impulse noise. The presence of noise in biomedical images such as MRI, X-ray, ECG, EEG etc can reduce their quality that complicates the diagnosis and treatment of diseases. Due to this, the restoration of noise-corrupted biomedical images has become a rapidly growing research interest in recent years. The pixels corrupted by impulse noise take the intensity values distinct from its neighboring uncorrupted pixels. Hence based on the properties of Impulse noise, it is classified into two main types such as, fixed-valued impulse noise that is also known as salt-and-pepper noise (SPN) and random-valued impulse noise (RVIN)(Chen \& $\mathrm{Wu}, 2001)$. Suppose a pixel corrupted with IN takes intensity values in two sets $s_{1}=\{0,1, \ldots, \gamma\}$ and $s_{2}=\{255-\gamma, 255-(\gamma-1), \ldots ., 255\}$ assuming $\gamma=0$ for an 8-bit gray scale image. For the images corrupted with SPN, the noisy pixels take a fixed 
intensity value distinct from its neighboring pixels that is either from the lower extreme or from the upper extreme values according to the histogram distribution of the gray level intensities of an image. Whereas in case of RVIN, corrupted pixels can take any intensity value ' $n_{i, j}$ ' ranging from ' $s_{1}$ ' to ' $s_{2}$ ' with un-equal probabilities. The probability mass function (pmf) for $S P N_{i, j}$ and $R V I N_{i, j}$ is expressed as follows:

$$
\begin{gathered}
\mathbf{S P N}_{i, j}=\left\{\begin{array}{c}
s_{1} \text { or } s_{2}, \text { with probability } p \\
L_{i, j}, \quad \text { with probability } 1-p
\end{array}\right. \\
\mathbf{R V I N}_{i, j}=\left\{\begin{array}{l}
n_{i, j}, \text { with } \text { probability } p \\
L_{i, j}, \text { with probability } 1-p
\end{array}\right.
\end{gathered}
$$

Where ' $n_{i, j}$ ' denotes gray level intensity of noisy pixel, ' $L_{i, j}$ ' represents the intensity value of noisefree pixels, while' $p$ ' is the probability of occurrence of noise in an image. Due to the uneven distribution of noisy pixels in case of RVIN, it is difficult to identify and restore them as compared to SPN. A pixel distinct from its neighboring pixels by taking a fixed intensity value is easy to identify as compared to a pixel that takes any intensity value from the entire range of gray level intensities present in an image. Furthermore for higher intensities of RVIN, the noisy pixels are also located in the neighborhood. Therefore, the intensity value of targeted noisy pixel might overlap with that of neighboring noisy pixels and hence the distinction among noisy and noise-free pixels becomes difficult and so is the identification. It leads to miss classification of noisy pixels that will increase the ratio of miss detections and false detections of noisy pixels at the noise identification stage.

\subsection{Motivation}

While restoring an image corrupted with RVIN, the texture and edge information must remain intact as the images are further used in various fields. In most cases, the distinction among noisy and edge pixels becomes difficult as the intensity values of some edge pixels might overlap with the noisy pixels' intensities. The noisy pixels exhibit an intensity value distinct from its neighboring pixels' intensities(Kang
\& Wang, 2009) however, some actual image's pixels such as edge pixels take intensity values different from some of its neighboring pixels' intensity(Toh \& Isa, 2010). Hence, it is identified as noisy and leads to inaccurate restoration of edge and the overall texture of an image. Moreover if the edge lies in some bright or dark area of image, the intensity value of the edge pixel is higher or lower than its neighboring pixels' intensities and hence, it is treated as noisy. Same is the case with noise filtering stage, some noisy pixels that are missed to identify as noisy in the noise identification stage might involve in the restoration of images that will lead to ineffective restoration. Cluster-based adaptive fuzzy switching median filter (CAFSM)(Toh \& Isa, 2010) that is designed on the basis of Rank-Order-AbsoluteDifferences (ROAD) can detect the impulses showing large variations. However, it did not work well for pixels showing little variation i.e. edge pixels. Some denoising techniques are presented to remove salt-and-pepper noise from X-ray and MRI biomedical images, respectively( $\mathrm{Lu}$ et al., 2018) (Murugan, Arunachalam, \& Karthik, 2018).

\subsection{Contribution}

To overcome the uncertainty encountered between an edge pixel and a noisy pixel and to better preserve the texture of restored images, the local information of texture must remain intact that will lead to its global texture preservation. To preserve the texture information locally, we propose to use textons in distinct orientations of the sliding window that preserves the edges and texture of images, effectively. Textons are formed by moving an isometric grid on the whole sliding window to cover every pixel of the sliding window, properly. It will lead to the effective preservation of texture of restored images. The aim of this paper is to propose a switching technique for the denoising of images corrupted with RVIN by using textons. The proposed denoising method first identifies the corrupted pixels by utilizing the local statistics of the textons in an iterative manner. Most similar tri-directional pixels across the central identified noisy pixel are employed to estimate the intensity values of corrupted pixels by using the fuzzy logics. Our major contribution in this paper is summarized as follows: 
- An iterative RVIN identification method is proposed that exploits the local statistics of the proposed textons by using an adaptive threshold range. Textons preserve the salient edges and texture of an image, effectively.

- An iterative two-phase noise filtering method is proposed by utilizing the most similar tri-directional pixels. In the first phase, fuzzy membership functions are employed to distinguish among noisy and noise-free pixels obtained from tridirectional pixels. In the second phase, most similar noise-free pixels are used to estimate the intensity values of identified noisy pixels by using fuzzy rules.

The remaining paper is organized in the following way: a comprehensive literature review is given in Sec. 2; Section 3 describes in detail the proposed RVIN denoising algorithm. The dataset used, the experiments conducted and the results gathered are presented and discussed in Sec. 4 whereas, a brief conclusion is given in Sec. 5 .

\section{Literature Review}

Numerous denoising methods have been introduced till now for the restoration of images corrupted with IN and RVIN. In the recent years, noise identifiers followed by noise filtering denoising methods have been proposed that performs better as compared to previous methods. The corrupted pixels are first identified by the noise identifier and then the filtering process is applied to restore the identified corrupted pixels. These methods are applied only to the noisy pixels to avoid the damage done to the noise-free pixels in methods having no noise identification stage. The most common among these two-stage denoising methods is Adaptive center-weighted median (ACWM) (Caiquan \& Dehua, 2008) filter that utilizes the adaptive thresholds mechanism at the noise identification stage. Modified progressive switching median filter (MPSM) (Kuykin, Khryashchev, \& Apalkov, 2009) was introduced that also works on the same mechanism. Directionalweighted-median (DWM) filter (Dong \& Xu, 2007) was proposed for the restoration of images corrupted with RVIN. In this, the RVIN identifier is based on the absolute difference among the pixels of the optimal directions in a sliding window. After the identification of corrupted pixels, these are restored by an adaptive weighted median filter that is an enhanced form of weighted median filter. The local statistics of the sliding window was also used for the denoising of images corrupted with RVIN by defining a pre-defined threshold value at the noise identification stage (Hassan Dawood, Dawood, \& Guo, 2015). The most similar pixels obtained from proposed optimal directions across the central pixel were chosen for the replacement of central identified noisy pixel. However, using a single threshold value may not correctly identify the noisy pixels as there can be many pixels greater than threshold value but are not noisy such as, edge pixel. This may lead to increase in the rate of miss detections and false detections of noisy pixels. An adaptive dual threshold median filter (ADTMF)(Gupta, Chaurasia, \& Shandilya, 2015) was proposed that identifies the corrupted pixels by comparing it with dual threshold values instead of a single threshold. It overcomes the drawbacks of noise identifiers having single threshold. This filter is effective for lower intensities of RVIN while it did not work well for the higher ones. A method for the restoration of images corrupted with RVIN was proposed that identifies the corrupted pixels using the information of neighboring pixels in multiple phases(Turkmen, 2013). Simple median filter was then used at the end of each phase for the restoration of identified corrupted pixels. The drawback of this approach is the increased computational cost due to multiple phases and the utilization of MF at the noise removal stage that treats noisy as well as noise-free pixels in similar manner. The weighted mean filter (L. Liu, Chen, Zhou, \& You, 2015) was designed using (RODROAD) that identifies the corrupted pixels in a two phase process and then filtered them using new WMF. This filter acts like an average filter and hence not suitable for impulse noise removal. A filter for the restoration of RVIN corrupted pixels was proposed that considers the most similar neighboring pixels for the identification of corrupted pixels (Habib, Rasheed, Hussain, \& Ali, 2015). Fuzzy rule based median filter was used at the noise removal stage. Local statistics based two stage adaptive rankordered impulse identifier was implemented $(\mathrm{Xu}, \mathrm{Li}$, Guo, Wu, \& Sbert, 2018) that can identify the RVIN corrupted pixels. The intensity values of identified 
corrupted pixels were estimated by combining edge preserving regularization (EPR) method at the noise filtering stage. It works in an iterative manner to identify most of the corrupted pixels, effectively. A universal denoising framework for the removal of impulse, Gaussian and impulse-Gaussian mixed noise was proposed (Xiong \& Yin, 2012). It was designed on the basis of non-local means (NLM) and robust outlyingness ratio (ROR) was used to identify the impulses. Modified directional weighted (MDW) filter (Li, Liu, Xu, \& Cheng, 2014) was implemented for the restoration of salt-and-pepper like impulses by considering the deficiencies found in DWM and MDWM filters.

Artificial intelligence based RVIN removal techniques including neural networks (NN) and fuzzy systems were also introduced in the last few years that have effective noise identification and removal capability as compared to other techniques. A nonlinear two-step noise removal technique (FIDRM) (Schulte, Nachtegael, De Witte, Van der Weken, \& Kerre, 2006) was used to remove IN while preserving the edges of an image. It uses a fuzzy logic approach at the noise identification stage that is basically a fuzzy average of pixels present in the neighborhood. Fuzzy random impulse noise reduction method (FRINRM) (Schulte, De Witte, Nachtegael, Van der Weken, \& Kerre, 2007) was utilized to remove RVIN from images that adopt a fuzzy logic approach at the noise identification and removal stage. Adaptive approaches for estimating thresholds were proposed with fuzzy logic approach at the noise filtering stage (Habib, Hussain, \& Choi, 2015)(Habib, Hussain, Rasheed, \& Ali, 2016). It gives better results at the regions of constant gray levels but produce blurry edges due to undiscriminating gray level intensities of noisy and edge pixels. Improved adaptive impulsive noise suppression method (IAINS)(Sa \& Majhi, 2010) was proposed to suppress noise from images corrupted with RVIN. However, the identifier proposed in it works well only for images corrupted with lower intensities of RVIN (i.e. up to $30 \%$ noise). A denoising method having noise identifier based on artificial neural network (ANN) and edge preserving regularization at the noise removal stage was presented (Turkmen, 2016) to identify and restore the RVIN corrupted pixels, respectively. This technique preserves the edges of restored images, effectively. A new clustering method using the directional neighbors for the suppression of lower as well as higher intensities of RVIN was proposed (Hussain \& Habib, 2017). Fuzzy logic approach was used at the noise identification stage while the filtration of noise is performed by median filter.

Recently, several image denoising techniques for the restoration of biomedical images corrupted with impulse noise were proposed. A directional weighted mean filter was employed for the denoising of X-ray bio images by using a block matching approach ( $\mathrm{Lu}$ et al., 2018). The technique is effective for the removal of SPN. An adaptive switching mean filter was designed for the denoising of electrocardiograms (ECG) by utilizing empirical mode decomposition (Rakshit \& Das, 2018). A multi-resolution hybrid filter for the restoration of MRI images corrupted with SPN was proposed (Murugan et al., 2018). It was implemented by integrating a dual-threshold median filter and a verge distance filter to get better quality of restored MRI images.

The concept of texton was introduced and it plays an important role in texture analysis (Julesz, 1981). Earlier, it was used in image retrieval (G.-H. Liu, Zhang, Hou, Li, \& Yang, 2010) where the images are retrieved on the basis of texture of images by proposing textons of specific structures. Recently, the concept of texton was introduced for denoising of images corrupted with RVIN in (Hussain Dawood, Dawood, \& Guo, 2017). Local statistics of the sliding window is used to identify the noisy pixels while multi-textons are used to restore the identified noisy pixels. Textons give sharp edges while restoring an image as texton itself is a gradient and hence, preserve the texture information of restored images. However, the textons proposed here consists of only three pixels that did not give best estimate for the intensity value of a noisy pixel, especially for higher intensities of RVIN. It is because all or most of the pixels in a texton might be noisy for higher noise densities and the noisy pixel may again get replaced by another noisy pixel. To overcome its drawback, a new texton-based image denoising technique was proposed (Azhar, Dawood, \& Dawood, 2018). This technique employs a $5 \times 5$ sliding window to extract textons of distinct orientations to preserve texture, effectively. The noise identifier proposed here works 
on the basis of multiple iterations. However, the drawback of this approach is its high computational cost due to extraction of large amount of textons as compared to the previous texton-based method and the executions of multiple iterations.

\section{Proposed RVIN denoising algorithm}

In corrupted images, edge or texture pixels are often misclassified as noisy pixels because the intensity values of some edge pixels are overlapping with noisy pixels' intensities. To handle the uncertainty between an edge pixel and a noisy pixel, we propose a switching denoising algorithm for the restoration of images corrupted with RVIN. It identifies the corrupted pixels in the first stage by utilizing the local statistics of textons and then estimates their intensity values at the noise filtering stage to restore the corrupted pixels as described in Sec. 3.1 and 3.2, respectively. Figure 1 shows the main steps of the proposed denoising algorithm:

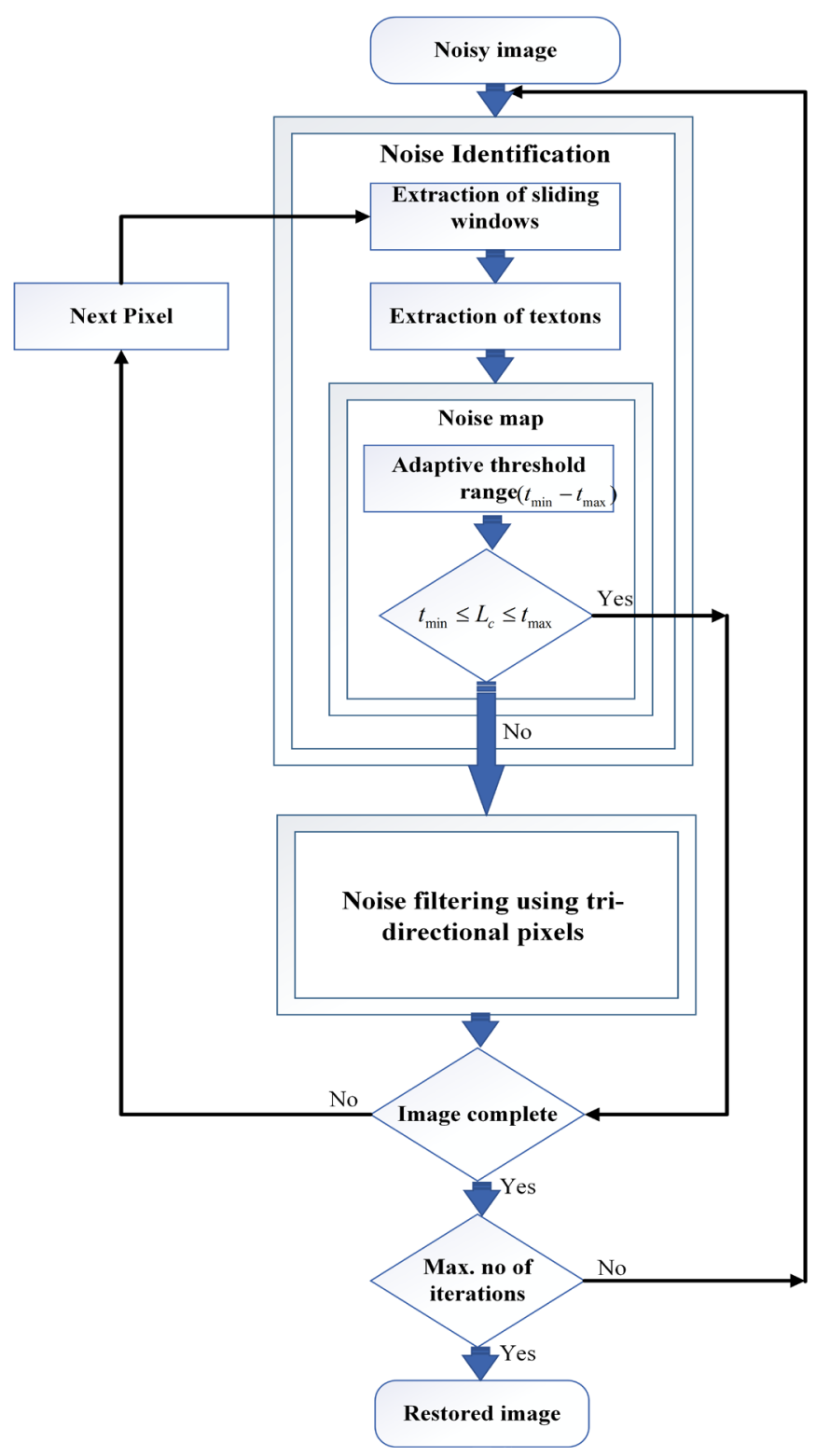

Fig. 1 Flow diagram of proposed RVIN denoising algorithm. 


\subsection{Noise Identification using textons}

Pixels corrupted with RVIN exhibit a slight difference from the neighboring noise-free pixels, especially for higher intensities of noise. So, the identification of corrupted pixels becomes difficult. It is because for higher noise intensities, the pixels in the neighborhood of the current noisy pixel are also corrupted and their intensity values are also lying in the range of noisy pixels' intensities. Furthermore, the distinction between an edge pixel and a noisy pixel is also difficult as the intensity values of edge pixels overlap with some of the neighboring noisy pixels' intensities. Therefore, it is necessary to adopt an effective RVIN identification technique that will lead to better estimation of intensity values of corrupted pixels at the noise filtering stage.

To deal with the problem of effective identification and the preservation of edge pixels, we propose
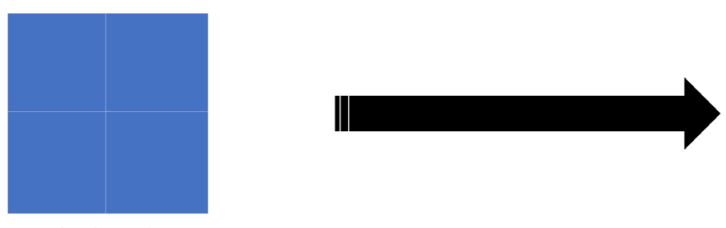

$2 \times 2$ grid textons in various orientations of the sliding window of size no larger than ' $S_{w} \times S_{w}$ ' where $\left\{S_{w} \in \square \mid 1 \leq S_{w} \leq 5\right\}$. Moreover, the proposed noise identifier works in an iterative manner to identify most of the noisy pixels by varying the estimated threshold range. Iterative nature of the proposed identifier ensures the effective identification of corrupted pixels as it identifies noisy pixels more than once. Hence, some of the noisy pixels that are left to be identified in earlier iterations will be identified in successive iterations that reduce the probability of miss detection and false detection. The orientations of textons are decided by moving an isometric grid of size no larger than $2 \times 2$ from leftto-right and top-to-bottom of the ' $S_{w} \times S_{w}$ ' with a step-length ' $S_{L}$ ' of one pixel. Consequently, the textons are formed along four horizontal directions ' $D_{H_{1}}{ }^{\prime}, D_{H_{2}}{ }^{\prime}, D_{H_{3}}$ ' and ' $D_{H_{4}}$ ' as shown in Fig. 2.

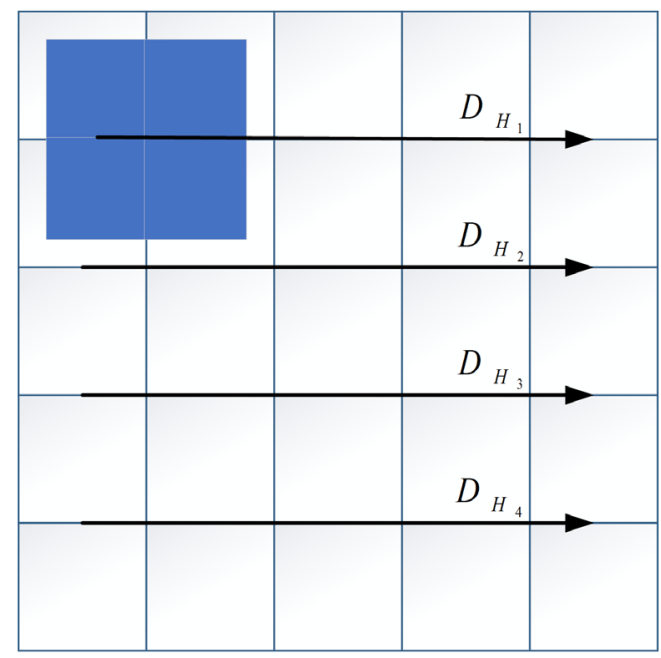

Textons Extraction

texture of image, both locally and globally. The central targeted pixel ' $L_{c}$ ' of ' $S_{w} \times S_{w}$ ' is included in each texton. The sequence for extracting textons is indicated in Eq. 2:

$\operatorname{Texton}\left(D_{H_{K}}\right)=\left\{L_{i}, L_{i+1}, L_{i+5}, L_{i+6}\right\}+L_{c}$

each pixel located at a specific position of a sliding window ' $S_{w} \times S_{w}$ '. As four textons are formed in each horizontal direction so, a total of sixteen textons 
are formed in a sliding window ' $S_{w} \times S_{w}$ ' as shown in Fig. 4. For each horizontal direction, the value of ' $i$ ' for Eq. 2 is chosen as indicated in Eq. 3(a-d):

$\mathbf{i}\left(D_{H_{1}}\right)=5 j-4, \forall j \in\{1,2,3,4\}$

$\mathbf{i}\left(D_{H_{2}}\right)=5 j-3, \forall j \in\{1,2,3,4\}$

$\mathbf{i}\left(D_{H_{3}}\right)=5 j-2, \forall j \in\{1,2,3,4\}$
$\mathbf{i}\left(D_{H_{4}}\right)=5 j-1, \forall j \in\{1,2,3,4\}$

' $j$ ' is a variable used to obtain the values of ' $i$ ' for each horizontal direction ' $D_{H_{K}}$ '.

The sliding window ' $S_{w} \times S_{w}$ ' and the proposed textons formed by using Eq. $2 \& 3$ are shown in Figs. 3 and 4, respectively:

\begin{tabular}{|c|c|c|c|c|}
\hline$L_{1}$ & $L_{2}$ & $L_{3}$ & $L_{4}$ & $L_{5}$ \\
\hline$L_{6}$ & $L_{7}$ & $L_{8}$ & $L_{9}$ & $L_{10}$ \\
\hline$L_{11}$ & $L_{12}$ & $L_{13}\left(L_{c}\right)$ & $L_{14}$ & $L_{15}$ \\
\hline$L_{16}$ & $L_{17}$ & $L_{18}$ & $L_{19}$ & $L_{20}$ \\
\hline$L_{21}$ & $L_{22}$ & $L_{23}$ & $L_{24}$ & $L_{25}$ \\
\hline
\end{tabular}

Fig. 3 Proposed sliding window.

After the extraction of textons, threshold range 'Thres_range' is determined from the pre-defined threshold value to identify the corrupted pixels. A pre-defined threshold value ' $T$ ' is set according to the intensities of noise. Single threshold value cannot identify all of the noisy pixels, effectively. It is because of the presence of edge pixels that may be identified as noisy for single threshold. Median is considered as the most appropriate measure for the estimation of threshold range. It is because median gives the intensity values that lie in the range of noise-free pixels and hence, the threshold range is computed, accurately. Therefore, the median ' $m$ ' of each individual texton is computed as shown in Eq. 4.

$\mathbf{m}=\forall$ Textons $\in S_{w} \times S_{w}$, median(Texton)

Consequently, sixteen median values i.e. ' $m_{1}, m_{2}, \ldots . ., m_{15}, m_{16}$ ' are obtained. Finally the median ' $M$ ' of median values ' $m$ ' computed in the previous step; is computed to get the value for defining the threshold range as indicated in Eq. 5.

$\mathbf{M}=\operatorname{median}(m)$

A threshold range ' $t_{\min }-t_{\max }$ ' is defined by incrementing or decrementing the value of predefined threshold ' $T$ ' by an amount of' $M$ '. Adding an increment in the value of' $T^{\prime}$, the highest graylevel intensity of threshold range ' $t_{\max }$ ' is obtained while ' $t_{\min }$ ' is obtained by decrementing it as indicated in the following equations:

Thres_range $=\mathbf{T} \pm M$

$\mathbf{t}_{\text {min }}=T-M$

$\mathbf{t}_{\max }=T+M$ 


\subsubsection{Noise map}

The proposed RVIN identification method works in an iterative manner by decrementing the pre-defined threshold value by a constant value of three. As a result, the estimated threshold range ' $t_{\min }-t_{\max }$ ' is varied in each iteration to identify the corrupted pixels that are left to be identified in earlier iterations. If the central targeted pixel ' $L_{c}$ ' of ' $S_{w} \times S_{w}$ 'lies within the threshold range ' $t_{\min }-t_{\max }$ ' in each iteration, it is labeled as noise-free as indicated in Eq.

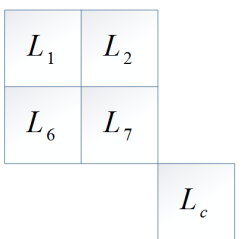

(a) Texton1.

\begin{tabular}{|l|l|}
\hline$L_{2}$ & $L_{3}$ \\
\hline$L_{7}$ & $L_{8}$ \\
\hline & $L_{c}$ \\
\hline
\end{tabular}

(e)Texton5.

\begin{tabular}{|l|l|}
\hline$L_{3}$ & $L_{4}$ \\
\hline$L_{8}$ & $L_{9}$ \\
\hline$L_{c}$ & \\
\hline
\end{tabular}

(i) Texton9.

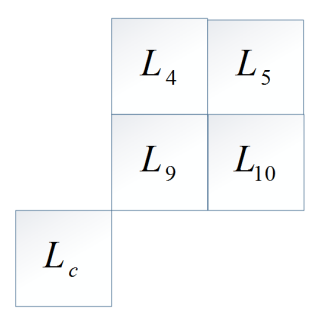

(m) Texton13.

\begin{tabular}{|l|l|l|}
\hline$L_{6}$ & $L_{7}$ & \\
\hline$L_{11}$ & $L_{12}$ & $L_{c}$ \\
\hline
\end{tabular}

(b) Texton2.

\begin{tabular}{|c|c|}
\hline$L_{7}$ & $L_{8}$ \\
\hline$L_{12}$ & $L_{13}+L_{c}$ \\
\hline
\end{tabular}

(f) Texton6.

\begin{tabular}{|c|c|}
\hline$L_{8}$ & $L_{9}$ \\
\hline$L_{13}+L_{c}$ & $L_{14}$ \\
\hline
\end{tabular}

(j) Texton10.

\begin{tabular}{|l|l|l|}
\hline \multicolumn{1}{|l|}{} & $L_{9}$ & $L_{10}$ \\
\hline$L_{c}$ & $L_{14}$ & $L_{15}$ \\
\hline
\end{tabular}

(n) Texton14.

$8 \mathrm{a}$ and is left without any further processing. On contrary to it, if it lies outside the threshold range in any iteration, it is considered as noisy as in Eq. 8b and is further processed by the proposed noise filtering method.

$\mathbf{L}_{c}$ (Noise_free $)=\left\{\begin{array}{l}1, t_{\min } \leq L_{c} \leq t_{\max } \\ 0, \quad \text { otherwise }\end{array}\right.$
$\mathbf{L}_{c}$ (Noisy) $= \begin{cases}1,\left(L_{c}<t_{\min }\right) & \text { OR } \quad \text { otherwise } \\ 0, & \left(L_{c}>t_{\max }\right)\end{cases}$

\begin{tabular}{|l|l|l|}
\hline$L_{11}$ & $L_{12}$ & $L_{c}$ \\
\hline$L_{16}$ & $L_{17}$ & \\
\hline
\end{tabular}

(c) Texton3.

\begin{tabular}{|l|l|}
\hline$L_{12}$ & $L_{13}+L_{c}$ \\
\hline$L_{17}$ & $L_{18}$ \\
\hline
\end{tabular}

(g) Texton7.

\begin{tabular}{|l|l|}
\hline$L_{13}+L_{c}$ & $L_{14}$ \\
\hline$L_{18}$ & $L_{19}$ \\
\hline
\end{tabular}

(k) Texton11.

\begin{tabular}{|l|l|l|}
\hline$L_{c}$ & $L_{14}$ & $L_{15}$ \\
\hline & $L_{19}$ & $L_{20}$ \\
\hline
\end{tabular}

(o) Texton15.

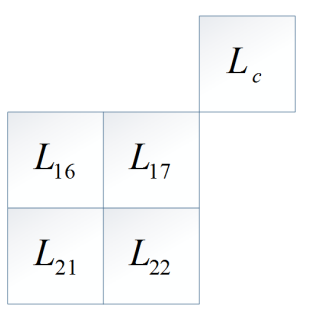

(d) Texton4.

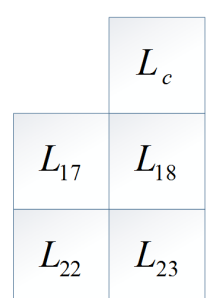

(h) Texton8.

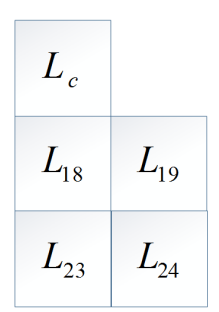

(I) Texton12.

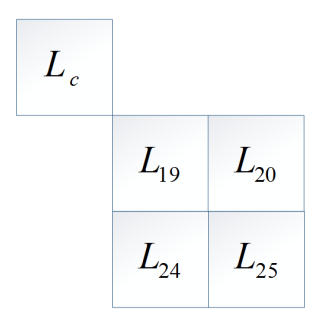

(p) Texton16.

Fig. 4 Proposed textons. 


\subsection{Noise filtering using tri-directional pixels}

It is essential to correctly estimate the intensity values of the identified noisy pixels to get the best approximate of original image. However, it is difficult especially for higher intensities of RVIN as the neighboring pixels may also get corrupted by noise in a random manner. Therefore, it is required to first identify whether the pixels used for the estimation of identified corrupted pixel are noise-free or not. As the pixels close to the central identified noisy pixel can give the best estimate of it so, three vertical directions ' $D_{1}{ }^{\prime}, D_{2}$ ' and ' $D_{3}$ ' in the immediate neighborhood of the central pixel ' $L_{c}$ ' are proposed. All pixels involved in the vertical directions are considered for the proposed noise filtering method except the central pixel ' $L_{c}$ ' as it is already identified as noisy. The vertical directional pixels used for the restoration of central noisy pixel are first identified as either corrupted by RVIN or not to avoid the noisy pixel to be replaced by another noisy pixel. Figure 5 shows the vertical directions considered for the restoration of noisy pixels. The shaded region shows the pixels involved in the proposed noise filtering method.

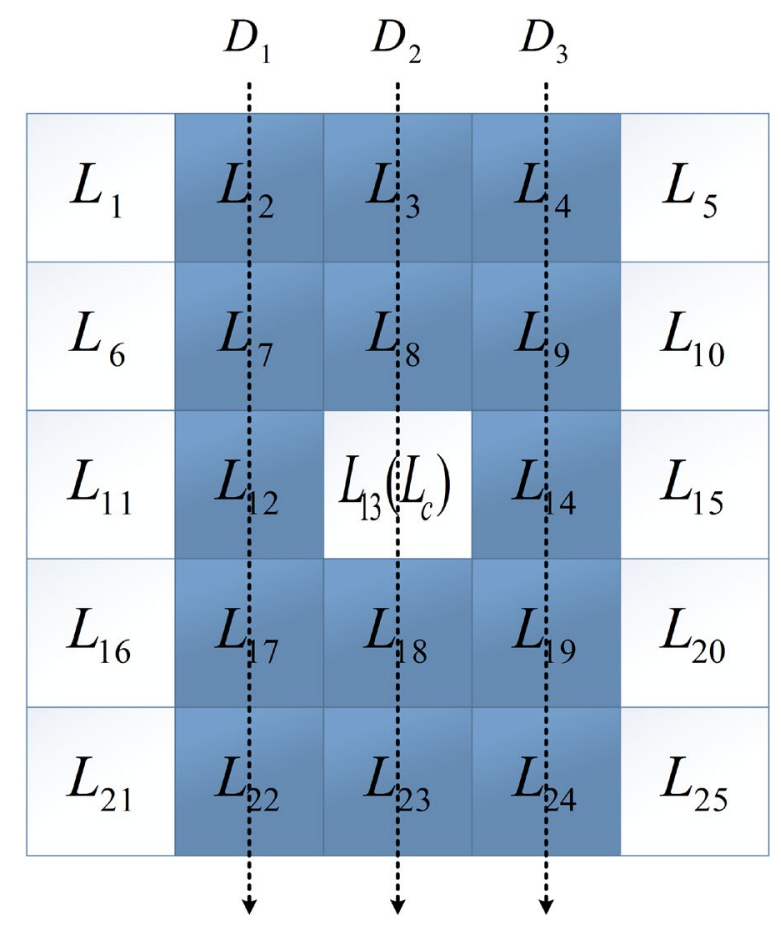

Fig. 5 Proposed vertical directions for noise filtering method.

The tri-directional pixels considered to estimate the intensity value of central noisy pixel are as follows:

$$
\begin{aligned}
& \mathbf{D}_{1}=\forall n \in\{0,1, \ldots, 4\}, L_{2+5 n} \\
& \mathbf{D}_{2}=\left(\forall n \in\{0,1, \ldots, 4\}, L_{3+5 n}\right)-L_{c} \\
& \mathbf{D}_{3}=\forall n \in\{0,1, \ldots, 4\}, L_{4+5 n}
\end{aligned}
$$

' $n$ ' is a variable used to obtain the gray level intensities of tri-directional pixels considered for the proposed noise filtration method. There might be a possibility of presence of some noisy pixels among them especially for higher intensities of RVIN as the noise is randomly distributed. Due to this, the restoration of noisy pixel will not be accurate. Therefore, we first estimate the relationship among the tri-directional pixels by applying fuzzy rules to separate the noise-free from noisy pixels. 


\subsubsection{Fuzzy membership functions for noise-free tri-directional pixels}

To obtain the noise-free pixels from the tridirectional pixels for the estimation of intensity value of noisy pixel, we propose fuzzy rules and fuzzy membership functions. Trapezoidal shaped fuzzy membership functions are proposed as they are capable to distinguish a noisy pixel from its neighboring noise-free pixels(Habib et al., 2016)(Schulte et al., 2007). Therefore, we propose two trapezoidal shaped fuzzy membership functions ' $N_{1}$ ' and ' $N_{2}$ ' as shown in Fig. 6. It has two rules ' $R_{1}$ ' and ' $R_{2}$ ' as the input is one i.e. ' $L_{k}$ ' and is expressed as follows:

$$
\begin{aligned}
& \mathbf{R}_{1}=N_{1}\left(L_{k}\right) \\
& \mathbf{R}_{2}=N_{2}\left(L_{k}\right)
\end{aligned}
$$

' $L_{k}{ }^{\prime}$ is the gray level intensity of tri-directional pixels involved in the proposed noise restoration method.

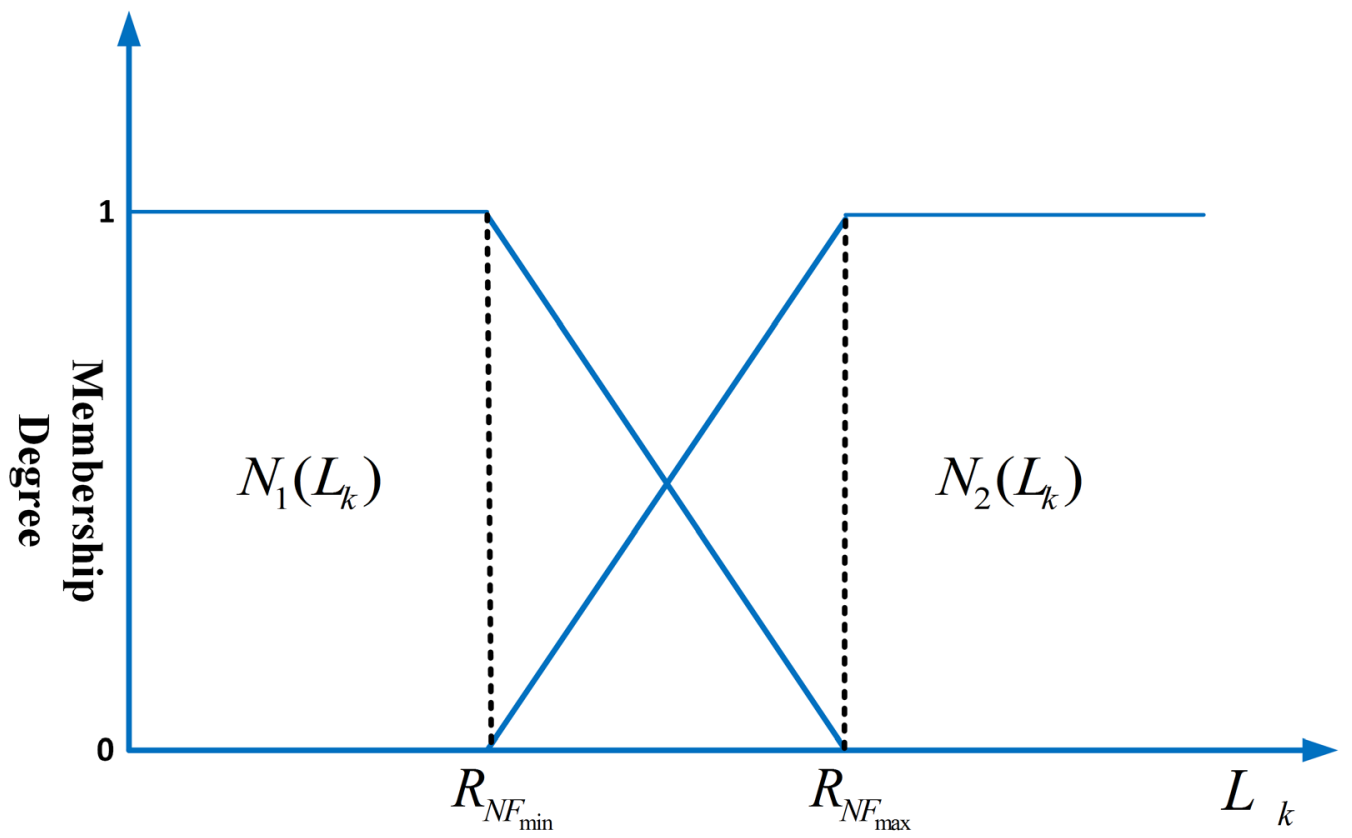

Fig.6 Trapezoidal-shaped fuzzy membership functions ' $N_{1}$ ' and ' $N_{2}$ '.

The trapezoidal shaped membership functions ' $N_{1}{ }^{\prime}$ and ' $N_{2}$ ' are expressed as in Eq. 11(a-b):

$$
\begin{aligned}
& \mathbf{N}_{1}\left(L_{k}\right)= \begin{cases}1, & L_{k}<R_{N F_{\min }} \\
S, R_{N F_{\min }} \leq L_{k} \leq R_{N F_{\max }} & L_{k}>R_{N F_{\max }}\end{cases} \\
& \mathbf{N}_{2}\left(L_{k}\right)= \begin{cases}1, & L_{k}>R_{N F_{\max }} \\
S, R_{N F_{\min }} \leq L_{k} \leq R_{N F_{\max }} & L_{k}<R_{N F_{\min }}\end{cases}
\end{aligned}
$$

Where, $S=\sum_{k=1}^{N}\left|L_{k}(i, j)-L_{k}(i, j+1)\right|$ is the sum of absolute inner differences of each pixel with all other pixels obtained in ' $N{ }^{\prime},{ }^{\prime} N$ ' is the total number of pixels obtained from tri-directional pixels satisfying the criteria for ' $S$ '. ' $R_{N F_{\min }}$ ' and ' $R_{N F_{\max }}$ 'are adaptive parameters discussed in Sec. 3.2.2.

\subsubsection{Fuzzy inference and adaptive parameters setting}

We propose an adaptive approach to adaptively set the parameters by using tri-directional pixels. To 
differentiate between noisy and noise-free pixels for the restoration of corrupted pixels, we used two parameters ' $R_{N F_{\min }}$ ' and ' $R_{N F_{\max }}$ '. The parameters can be expressed as in Eq.12:

$$
\begin{aligned}
& \mathbf{R}_{N F_{\min }}=\text { Med }- \text { constant_value } \\
& \mathbf{R}_{N F_{\max }}=\text { Med }+ \text { constant_value }
\end{aligned}
$$

Where,

$$
\operatorname{Med}=\forall \mathbf{L}_{k} \in D_{x}, \text { median }\left(L_{k}\right): x \in\{1,2,3\}
$$

Where ' $k$ ' represents the total number of tridirectional pixels. ' $D$ ' stands for the vertical directions considered for the noise filtration method. ' $L$ ' represents the gray level intensities of pixels involved in proposed vertical directions. We chose twenty-five as the most suitable constant_value for defining the range for noise-free pixels. To restore the pixels identified as noisy in Sec. 3.1, we use the following set of fuzzy rules to obtain noise-free pixels from the pixels obtained from fuzzy membership functions defined in Sec. 3.2.1:

$$
\mathbf{S}_{\text {small }} \Rightarrow \text { Noise_free }\left(L_{k}\right)
$$

$\mathbf{S}_{l \text { arge }} \Rightarrow \operatorname{Noisy}\left(L_{k}\right)$

' $S_{\text {small }}$ ' represents the smaller values of ' $S$ ', while ' $S_{l \text { arge }}$ ' stands for the largest values of ' $S$ '. Smaller the sum of absolute inner difference, greater will be the similarity. Therefore, the pixels having intensity values ' $L_{k}$ ' that give small values of ' $S$ ' indicated as ' $S_{\text {small }}$ ' are more similar to each other and are considered for the restoration of corrupted pixels as expressed in Eq. 15.

$\mathbf{L}_{s}=\forall n \in S_{\text {small }}, \operatorname{median}\left(L_{k} \square n\right)$

Where ' $L_{s}$ ' is considered as the most suitable value to estimate the gray level intensity value of noisy pixels for their restoration. ' $n$ ' is the total number of elements obtained in ' $S_{\text {small }}$ 'i.e. having smaller sum of absolute inner differences.
The algorithm for the proposed RVIN denoising algorithm is as follows:

Input: Image corrupted with RVIN.

Output: Restored image.

- A sliding window ' $S_{w} \times S_{w}$ ' is mapped over the whole input image.

- Extract the proposed textons from the sliding windows using Eq. 2.

- Estimate the threshold range from the predefined threshold value using Eq. 6 to identify the central targeted pixel of the sliding window as either corrupted with RVIN or not.

- If the targeted pixel is found corrupted, restore it by estimating its gray level intensity value using Eq. 15, otherwise left it as it is and move towards the next pixel.

Repeat all the steps till maximum allowable iterations completed.

\section{Experiments}

\subsection{Datasets and evaluation criteria}

The proposed denoising algorithm works on noise identification followed by noise filtering stage. The noise identifier plays a fundamental role in the overall denoising performance of the proposed algorithm. Therefore the performance of proposed noise identifier is first evaluated and then of noise filtering in Sec. 4.2.1 and 4.2.2, respectively. Evaluation metrics such as Peak-signal-to-noise ratio (PSNR) in decibel (dB)(Xiong \& Yin, 2012), Structural similarity index measurement (SSIM)(Wang, Bovik, Sheikh, \& Simoncelli, 2004), Miss detection (MD) and False detection (FD) are used to assess the overall performance of proposed image denoising algorithm. For quantitative and visual comparisons of proposed denoising algorithm with state-of-the-art methods, we divide test images in three main categories to show the significant performance of proposed method over a wide range of images. In the first category, seven standard benchmark test images including Lena, Bridge, Boat, Pentagon, Peppers, Goldhill and Baboon are used as shown in Fig. 7. All of these images are 8-bit 
grayscale images and have a spatial resolution of $512 \times 512$. In the second category, images randomly selected from USC-SIPI database located at http://sipi.usc.edu/services/database/Database.html are used. It consists of four volumes named as 'Textures', 'Aerials', 'Miscellaneous' and 'Sequences' of images of various sizes and dimensions. We used 'Miscellaneous' volume of USC-SIPI database to evaluate the performance of our algorithm. Some visual results on various images selected from USC-SIPI database are shown to evaluate the denoising and texture-preserving capability of the proposed algorithm. In the third category, the biomedical images selected from MedPix database are used to show the restoration results of the proposed algorithm on biomedical images. MedPix consists of nine main categories of biomedical images while, we used some images chosen from CT-Scan, MRI and X-ray to test the proposed algorithm. Furthermore, the settings of various parameters used and the computational cost for the proposed method are discussed in Sec. 4.2.4 and 4.2.5, respectively.

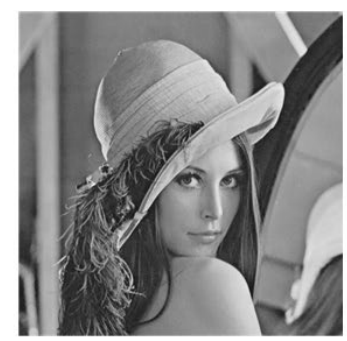

a) Lena.

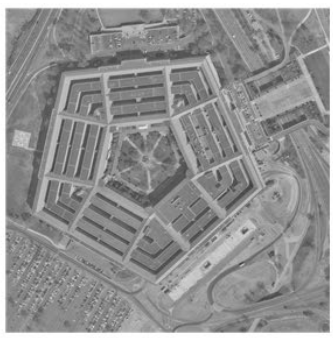

d) Pentagon.

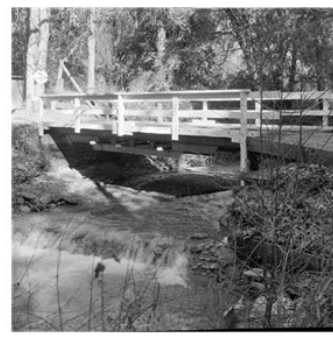

b) Bridge.

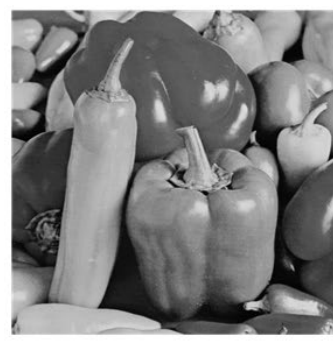

e) Peppers.

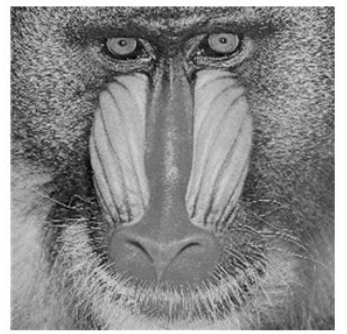

g) Baboon.

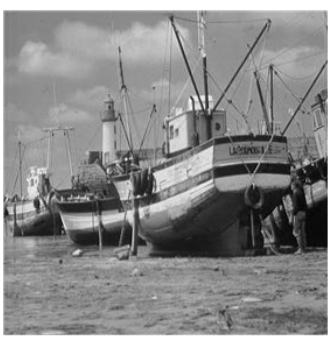

c) Boat.

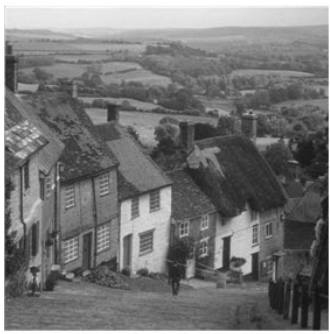

f) Goldhill.

Fig.7 Standard test images.

\subsection{Results and discussion}

To evaluate the performance of proposed image denoising algorithm, various state-of-the-art denoising methods such as ACWM(Caiquan \& Dehua, 2008), MPSM(Kuykin et al., 2009), FIDRM(Schulte et al., 2006), DWM(Dong \& Xu, 2007), FRINRM(Schulte et al., 2007), IAINS(Sa \&
Majhi, 2010), LNI-MTR(Hussain Dawood et al., 2017), ANN(Turkmen, 2016), MSN(Habib, Rasheed, et al., 2015), $\operatorname{ADTMF(Gupta~et~al.,~2015),~}$ CAFSM(Toh \& Isa, 2010), AFIDM(Habib et al., 2016), NLM(Xiong \& Yin, 2012), ATFDF(Habib, Hussain, et al., 2015), FPDM(Turkmen, 2013), MDW(Li et al., 2014), NWM(L. Liu, Chen, Zhou, \& You, 2015), DROAD(Hussain \& Habib, 2017) and 
TOID (Azhar et al., 2018) are used. Some of the methods are used to evaluate the noise identifier's

\subsubsection{Performance evaluation of noise identifier}

The effectiveness of a noise identifier is assessed on the amount of corrupted pixels it identifies correctly. Therefore, the performance of the proposed noise identifier is evaluated on the basis of miss detections (MD) and false detections (FD). MD refers to the amount of noisy pixels that are missed to be identified as either corrupted or not by the noise identifier, whereas; FD indicates the number of noise-free pixels that are incorrectly identified as noisy. Both MD and FD show the miss classification of pixels as either lying in the category of noisy or noise-free pixels. We used ACWM(Caiquan \& Dehua, 2008), MPSM(Kuykin et al., 2009), FIDRM(Schulte et al., 2006), DWM(Dong \& Xu, 2007), FRINRM(Schulte et al., 2007), IAINS(Sa \& Majhi, 2010), LNI-MTR(Hussain Dawood et al., 2017) and ANN(Turkmen, 2016) on standard Peppers, Goldhill and Lena images corrupted with various intensities of noise, to calculate the amount of miss detections and false detections for the proposed noise identifier. The comparison of MD and FD with state-of-the-art methods having noise identifiers are shown in Fig.8-10. It is clear from the graphs shown performance while others are used for the noise filtering's performance.

that the proposed noise identification method gives less number of MD and FD as compared to other methods. For some existing methods, its amount is low as compared to proposed method as shown graphically. However on average, proposed noise identifier gives better results with respect to MD and FD as compared to other methods. The better performance of proposed noise identifier is due to the orientations of textons proposed, the threshold range selected and the iterative nature of proposed noise identifier. The textons are proposed in such orientations that cover all pixels of the sliding window, properly. Due to this, the threshold range is computed by using the local statistics of textons, accurately. Local statistics of the textons give better estimate of the threshold range as compared to single threshold value. Moreover, the iterative nature of the noise identifier is another factor in the effective identification of corrupted pixels. As the proposed noise identifier identifies the noisy pixels in multiple iterations, therefore; the pixels that are left to be identified in earlier iterations are identified in upcoming iterations.

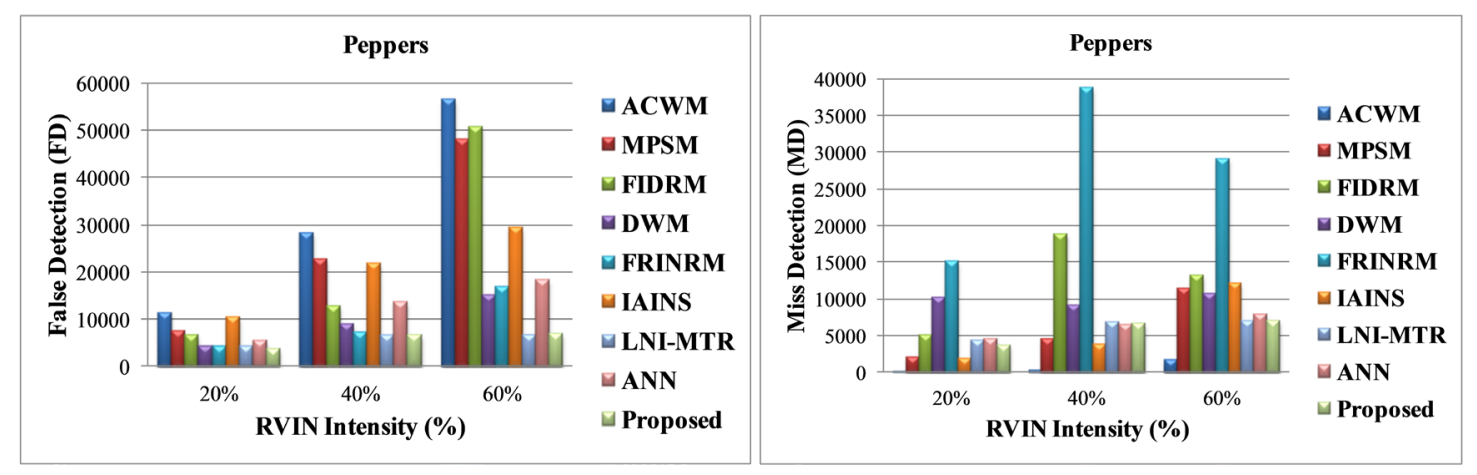

Fig.8 Graphical comparison of proposed noise identifier on Peppers image corrupted with various intensities of RVIN. 

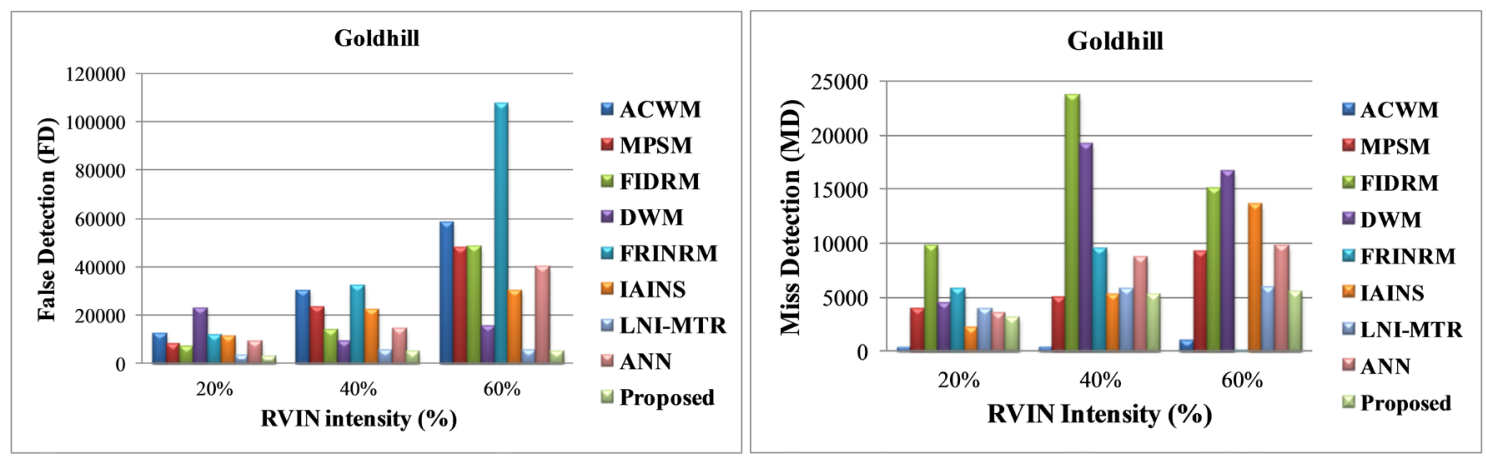

Fig.9 Graphical comparison of proposed noise identifier on Goldhill image corrupted with various intensities of RVIN.
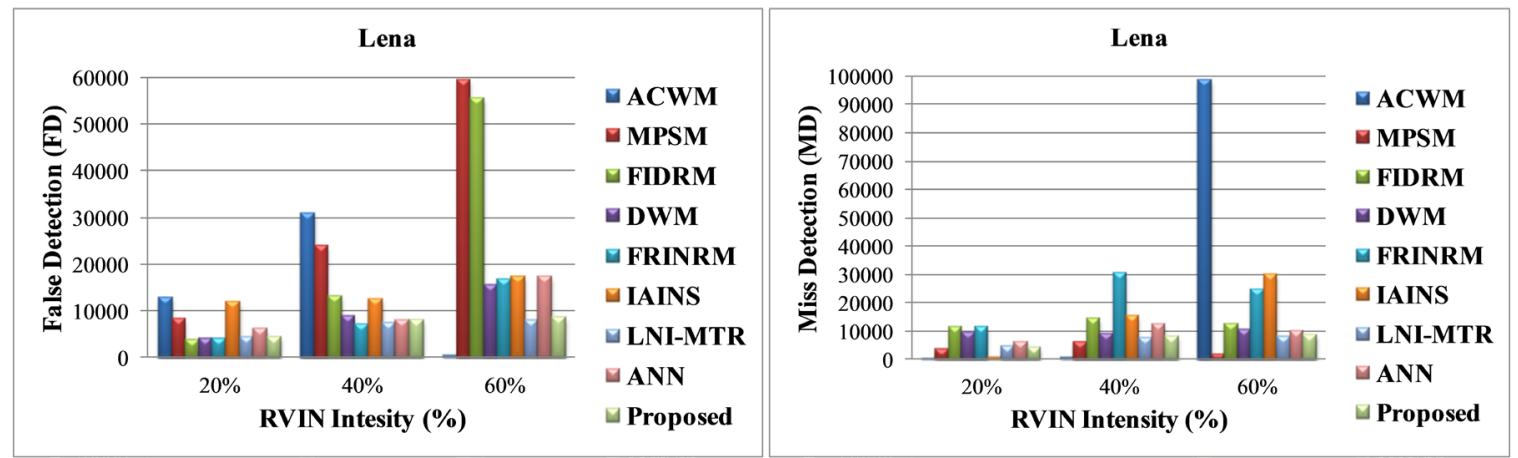

Fig.10 Graphical comparison of proposed noise identifier on Lena image corrupted with various intensities of RVIN.

\subsubsection{Performance evaluation of overall denoising method}

To evaluate the overall denoising performance of the proposed algorithm after applying proposed noise identification and filtering method, PSNR and SSIM are used. PSNR indicates the denoising capability of the proposed method and is expressed as follows:

$$
\text { PSNR }=\operatorname{10}_{\log }\left(\frac{M A X^{2}}{M S E}\right)
$$

Where ' $M A X^{\prime}$ represents the largest possible gray level intensity among the entire range of gray levels in accordance to the histogram distribution of the gray levels intensities present in an image. ' $M S E$ ' is the mean squared error between original image ' $O$ ' and restored image ' $R$ ' and is given by using Eq. 17:

$$
\text { MSE }=\left(\frac{1}{M \times N}\right) \sum_{i=1}^{M} \sum_{j=1}^{N}(O-R)^{2}
$$

Where ' $M \times N$ ' is the spatial resolution of original and restored images that should be same to compute 'MSE'. ' $M$ ' and ' $N$ ' represents the total number of rows and columns of an image, respectively. The other parameter used to assess the denoising performance is SSIM that is a measure to compute the degree of similarity between original noise-free and restored image and is expressed as:

$\operatorname{SSIM}=\frac{\left(2 \mu_{o} \mu_{R}+c_{1}\right)\left(2 \sigma_{O R}+c_{2}\right)}{\left(\mu_{o}^{2}+\mu_{R}^{2}\right)\left(\sigma_{o}^{2}+\sigma_{R}^{2}+c_{2}\right)}$

' $\mu_{O}{ }^{\prime},{ }^{\prime} \mu_{R}{ }^{\prime},{ }^{\prime} \sigma_{O}{ }^{\prime},{ }^{\prime} \sigma_{R}$ ' and ' $\sigma_{O R}$ ' stands for the means, variances and co-variances of original and restored images, respectively. ' $c_{1}$ ' and ' $c_{2}$ ' are some constant values.

The proposed denoising algorithm is compared with state-of-the-art denoising methods such as MSN(Habib, Rasheed, et al., 2015), ADTMF(Gupta et al., 2015), $\operatorname{CAFSM(Toh~\& ~Isa,~2010),~}$ 
AFIDM(Habib et al., 2016), NLM(Xiong \& Yin, 2012), ATFDF(Habib, Hussain, et al., 2015), FPDM(Turkmen, 2013), MDW(Li et al., 2014), NWM(L. Liu et al., 2015) LNI-MTR(Hussain Dawood et al., 2017), DROAD(Hussain \& Habib, 2017) and TOID(Azhar, Dawood, \& Dawood, 2018); to evaluate its restoration capability, both quantitatively and visually. The quantitative comparison of proposed denoising method with stateof-the-art methods is given in Tables (1-4) on different standard images based on PSNR and SSIM. The standard images taken for the comparison are corrupted with RVIN of intensities ranging from $10 \%$ to $50 \%$. The quantitative results of the proposed method show the superior performance of it by showing a considerable increase in the values of PSNR and SSIM on different images. The proposed denoising method gives increased values of PSNR and SSIM except for one case of SSIM, where its value is slightly less than the highest value. It indicates the improved restoration and denoising capability of the proposed method as compared to state-of-the-art methods. Moreover, the rate of fall in the values of PSNR and SSIM as the intensity of noise increases is low (i.e. just one or two points) that indicates the improved performance of proposed method for various intensities of RVIN. Quantitative results of the proposed denoising method are given in Table 5 on ten images of different sizes and spatial resolution randomly selected from USC-SIPI database. The colored images among them are first converted into grayscale to evaluate the denoising performance of the proposed algorithm. The average values of PSNR, SSIM, MD and FD shown in Table 5 indicate that the rate of fall in their values is in uniform manner. It indicates that the proposed method performs well for standard as well as other types of images for both noise identification and filtering.

Table 1 Comparison of PSNR (dB) and SSIM values of proposed and state-of-the-art denoising methods on Lena image corrupted with various intensities of RVIN.

\begin{tabular}{|c|c|c|c|c|c|c|c|c|c|c|}
\hline \multirow{3}{*}{ Methods } & \multicolumn{10}{|c|}{ RVIN intensity } \\
\hline & \multicolumn{2}{|c|}{$10 \%$} & \multicolumn{2}{|c|}{$20 \%$} & \multicolumn{2}{|c|}{$30 \%$} & \multicolumn{2}{|c|}{$40 \%$} & \multicolumn{2}{|c|}{$50 \%$} \\
\hline & PSNR & SSIM & PSNR & SSIM & PSNR & SSIM & PSNR & SSIM & PSNR & SSIM \\
\hline $\begin{array}{l}\text { MSN(Habib, Rasheed, et } \\
\text { al., 2015) }\end{array}$ & 39.62 & 0.9623 & 38.43 & 0.9253 & 36.79 & 0.9127 & 34.92 & 0.8998 & 32.45 & 0.8712 \\
\hline $\begin{array}{c}\text { ADTMF(Gupta et al., } \\
2015)\end{array}$ & 41.02 & 0.9694 & 38.09 & 0.9487 & 34.67 & 0.9314 & 31.45 & 0.9221 & 29.15 & 0.8906 \\
\hline CAFSM(Toh \& Isa, 2010) & 36.86 & 0.9612 & 35.45 & 0.9472 & 34.14 & 0.8939 & 32.87 & 0.8498 & 30.62 & 0.8719 \\
\hline $\operatorname{AFIDM(Habib~et~al.,~2016)~}$ & 38.85 & 0.9583 & 37.92 & 0.9317 & 36.39 & 0.9112 & 35.13 & 0.8804 & 33.69 & 0.8463 \\
\hline NLM(Xiong \& Yin, 2012) & 38.50 & 0.9690 & 36.83 & 0.9507 & 34.55 & 0.9328 & 32.96 & 0.9219 & 30.79 & 0.8935 \\
\hline $\begin{array}{c}\text { ATFDF(Habib, Hussain, et } \\
\text { al., 2015) }\end{array}$ & 38.10 & 0.9672 & 37.62 & 0.9518 & 36.2 & 00 & 35.08 & 0.9098 & 33.37 & 0.8867 \\
\hline FPDM(Turkmen, 2013) & 36.23 & 0.9462 & 35.45 & 0.9373 & 33.93 & 0.9127 & 32.59 & 0.8973 & 30.75 & 0.8634 \\
\hline MDW(Li et al., 2014) & 38.9 & 0.9509 & 36.30 & 0.9390 & 34.00 & 0.9218 & 32.09 & 0.9163 & 30.20 & 0.8851 \\
\hline NWM(L. Liu et al., 2015) & 38.87 & 0.9697 & 36.23 & 0.9529 & 34.99 & 0.9314 & 33.73 & 0.9219 & 31.80 & 0.9009 \\
\hline $\begin{array}{l}\text { LNI-MTR(Hussain } \\
\text { Dawood et al., 2017) }\end{array}$ & 41.99 & 0.9709 & 38.81 & 0.8803 & 36.42 & 0.7486 & 34.66 & 0.5787 & 33.16 & 0.4361 \\
\hline $\begin{array}{l}\text { DROAD(Hussain \& Habib, } \\
\text { 2017) }\end{array}$ & 41.29 & 0.9743 & 39.38 & 0.9541 & 37.11 & 0.9358 & 35.50 & 0.9233 & 34.22 & 0.8997 \\
\hline TOID(Azhar et al., 2018) & 41.49 & 0.9730 & 39.06 & 0.9610 & 37.75 & 0.9441 & 36.45 & 0.858 & 34.91 & 0.8045 \\
\hline Proposed & 42.86 & 0.9890 & 40.24 & 0.9784 & 38.64 & 0.9673 & 37.61 & 0.9541 & 36.89 & 0.9286 \\
\hline
\end{tabular}


Table 2 Comparison of PSNR (dB) and SSIM values of proposed and state-of-the-art denoising methods on Bridge image corrupted with various intensities of RVIN.

\begin{tabular}{|c|c|c|c|c|c|c|c|c|c|c|}
\hline \multirow{3}{*}{ Methods } & \multicolumn{10}{|c|}{ RVIN intensity } \\
\hline & \multicolumn{2}{|c|}{$10 \%$} & \multicolumn{2}{|c|}{$20 \%$} & \multicolumn{2}{|c|}{$30 \%$} & \multicolumn{2}{|c|}{$40 \%$} & \multicolumn{2}{|c|}{$50 \%$} \\
\hline & PSNR & SSIM & PSNR & SSIM & PSNR & SSIM & PSNR & SSIM & PSNR & SSIM \\
\hline $\begin{array}{c}\text { MSN(Habib, Rasheed, et } \\
\text { al., 2015) }\end{array}$ & 30.62 & 0.8987 & 29.71 & 0.8654 & 28.08 & 0.8200 & 26.06 & 0.7338 & 23.99 & 0.6746 \\
\hline $\begin{array}{c}\text { ADTMF(Gupta et al., } \\
2015)\end{array}$ & 31.00 & 0.8965 & 29.81 & 0.8282 & 27.17 & 0.7543 & 25.65 & 0.6962 & 23.11 & 0.5862 \\
\hline CAFSM(Toh \& Isa, 2010) & 27.94 & 0.8915 & 26.77 & 0.8420 & 25.54 & 0.7972 & 24.19 & 0.7446 & 22.95 & 0.6892 \\
\hline $\begin{array}{c}\operatorname{AFIDM}(\text { Habib et al., } \\
2016)\end{array}$ & 29.05 & 0.9250 & 28.11 & 0.8908 & 26.79 & 0.8322 & 25.82 & 0.7499 & 24.35 & 0.7076 \\
\hline NLM(Xiong \& Yin, 2012) & 28.63 & 0.8981 & 27.34 & 0.8557 & 26.03 & 0.8093 & 24.82 & 0.7201 & 23.07 & 0.6470 \\
\hline $\begin{array}{c}\text { ATFDF(Habib, Hussain, } \\
\text { et al., 2015) }\end{array}$ & 30.80 & 0.9154 & 29.19 & 0.8836 & 27.82 & 0.8373 & 25.52 & 0.7469 & 24.00 & 0.6990 \\
\hline FPDM(Turkmen, 2013) & 29.44 & 0.9021 & 28.25 & 0.8289 & 27.18 & 0.7709 & 25.40 & 0.7222 & 23.96 & 0.6510 \\
\hline MDW(Li et al., 2014) & 29.02 & 0.8999 & 27.79 & 0.8264 & 25.33 & 0.7945 & 23.66 & 0.7169 & 22.58 & 0.6419 \\
\hline NWM(L. Liu et al., 2015) & 30.97 & 0.9155 & 28.79 & 0.8899 & 26.92 & 0.8082 & 24.80 & 0.7488 & 23.69 & 0.7047 \\
\hline $\begin{array}{l}\text { LNI-MTR(Hussain } \\
\text { Dawood et al., 2017) }\end{array}$ & 34.32 & 0.9284 & 33.60 & 0.8664 & 32.88 & 0.7624 & 32.09 & 0.6404 & 31.36 & 0.5040 \\
\hline $\begin{array}{l}\text { DROAD(Hussain \& } \\
\text { Habib, 2017) }\end{array}$ & 31.30 & 0.9266 & 30.10 & 0.8975 & 28.72 & 0.8436 & 26.10 & 0.7575 & 25.35 & 0.7108 \\
\hline TOID(Azhar et al., 2018) & 34.97 & 0.9011 & 34.08 & 0.9350 & 32.72 & 0.8650 & 31.77 & 0.7830 & 30.96 & 0.7132 \\
\hline Proposed & 37.24 & 0.9495 & 36.01 & 0.9324 & 34.86 & 0.9029 & 33.63 & 0.8554 & 32.60 & 0.7875 \\
\hline
\end{tabular}

Table 3 Comparison of PSNR (dB) and SSIM values of proposed and state-of-the-art denoising methods on Boat image corrupted with various intensities of RVIN.

\begin{tabular}{|c|c|c|c|c|c|c|c|c|c|c|}
\hline \multirow{3}{*}{ Methods } & \multicolumn{10}{|c|}{ RVIN intensity } \\
\hline & \multicolumn{2}{|c|}{$10 \%$} & \multicolumn{2}{|c|}{$20 \%$} & \multicolumn{2}{|c|}{$30 \%$} & \multicolumn{2}{|c|}{$40 \%$} & \multicolumn{2}{|c|}{$50 \%$} \\
\hline & PSNR & SSIM & PSNR & SSIM & PSNR & SSIM & PSNR & SSIM & PSNR & SSIM \\
\hline $\begin{array}{l}\text { MSN(Habib, Rasheed, et } \\
\text { al., 2015) }\end{array}$ & 34.98 & 0.9115 & 33.71 & 0.8802 & 32.12 & 0.8561 & 30.00 & 0.8177 & 27.82 & 0.7720 \\
\hline $\begin{array}{l}\text { ADTMF(Gupta et al., } \\
\text { 2015) }\end{array}$ & 35.10 & 0.9396 & 33.98 & 0.8908 & 32.01 & 0.8456 & 29.99 & 0.8190 & 26.65 & 0.7321 \\
\hline $\begin{array}{c}\text { CAFSM(Toh \& Isa, } \\
2010)\end{array}$ & 32.92 & 0.9283 & 31.19 & 0.9189 & 29.69 & 0.8544 & 27.30 & 0.8064 & 25.88 & 0.7483 \\
\hline $\begin{array}{l}\text { AFIDM(Habib et al., } \\
\text { 2016) }\end{array}$ & 33.51 & 0.9385 & 32.29 & 0.8980 & 31.07 & 0.8547 & 29.00 & 0.8094 & 28.42 & 0.7684 \\
\hline $\begin{array}{c}\text { NLM(Xiong \& Yin, } \\
\text { 2012) }\end{array}$ & 32.46 & 0.9345 & 31.12 & 0.9164 & 29.75 & 0.8539 & 27.48 & 0.8318 & 25.99 & 0.7806 \\
\hline $\begin{array}{c}\text { ATFDF(Habib, Hussain, } \\
\text { et al., 2015) }\end{array}$ & 34.73 & 0.9388 & 33.68 & 0.9193 & 31.89 & 0.8499 & 29.22 & 0.8187 & 28.31 & 0.7701 \\
\hline FPDM(Turkmen, 2013) & 33.45 & 0.9209 & 32.20 & 0.8920 & 30.92 & 0.8354 & 29.00 & 0.7865 & 26.76 & 0.7246 \\
\hline MDW(Li et al., 2014) & 33.22 & 0.9109 & 32.06 & 0.8912 & 30.82 & 0.8498 & 27.95 & 0.7929 & 26.50 & 0.7546 \\
\hline $\mathbf{N W M ( L . ~ L i u ~ e t ~ a l . , ~ 2 0 1 5 ) ~}$ & 34.90 & 0.9387 & 32.48 & 0.9155 & 30.69 & 0.8531 & 27.72 & 0.8308 & 26.45 & 0.7913 \\
\hline $\begin{array}{l}\text { LNI-MTR(Hussain } \\
\text { Dawood et al., 2017) }\end{array}$ & 33.2 & 0.9469 & 32.14 & 0.8685 & 34.40 & 0.7528 & 33.11 & 0.6003 & 31.87 & 0.4702 \\
\hline $\begin{array}{l}\text { DROAD(Hussain \& } \\
\text { Habib, 2017) }\end{array}$ & 35.76 & 0.9450 & 34.35 & 0.9211 & 32.46 & 0.8570 & 30.34 & 0.8329 & 29.43 & 0.7997 \\
\hline TOID(Azhar et al., 2018) & 35.92 & 0.9489 & 36.14 & 0.9201 & 32.81 & 0.9154 & 33.49 & 0.8623 & 32.29 & 0.7671 \\
\hline Proposed & 37.91 & 0.9756 & 38.63 & 0.9575 & 34.91 & 0.9345 & 35.75 & 0.9040 & 34.79 & 0.8551 \\
\hline
\end{tabular}


Table 4 Comparison of PSNR (dB) and SSIM values of proposed and state-of-the-art denoising methods on Pentagon image corrupted with various intensities of RVIN.

\begin{tabular}{|c|c|c|c|c|c|c|c|c|c|c|}
\hline \multirow{3}{*}{ Methods } & \multicolumn{10}{|c|}{ RVIN intensity } \\
\hline & \multicolumn{2}{|c|}{$10 \%$} & \multicolumn{2}{|c|}{$20 \%$} & \multicolumn{2}{|c|}{$30 \%$} & \multicolumn{2}{|c|}{$40 \%$} & \multicolumn{2}{|c|}{$50 \%$} \\
\hline & PSNR & SSIM & PSNR & SSIM & PSNR & SSIM & PSNR & SSIM & PSNR & SSIM \\
\hline $\begin{array}{l}\text { MSN(Habib, Rasheed, et } \\
\text { al., 2015) }\end{array}$ & 33.23 & 0.9334 & 32.16 & 0.9222 & 30.83 & 0.9101 & 28.04 & 0.8921 & 25.91 & 0.8676 \\
\hline $\begin{array}{l}\text { ADTMF(Gupta et al., } \\
\text { 2015) }\end{array}$ & 33.30 & 0.9102 & 32.12 & 0.8935 & 30.34 & 0.8456 & 28.09 & 0.7721 & 26.07 & 0.6872 \\
\hline $\begin{array}{c}\text { CAFSM(Toh \& Isa, } \\
2010)\end{array}$ & 30.72 & 0.9110 & 29.64 & 0.8786 & 28.43 & 0.8352 & 27.25 & 0.7793 & 26.05 & 0.7082 \\
\hline $\begin{array}{c}\operatorname{AFIDM}(\text { Habib et al., } \\
2016)\end{array}$ & 32.83 & 0.9522 & 31.79 & 0.9474 & 30.80 & 0.9387 & 30.05 & 0.9164 & 29.11 & 0.8949 \\
\hline $\begin{array}{c}\text { NLM(Xiong \& Yin, } \\
\text { 2012) }\end{array}$ & 31.56 & 0.9043 & 30.45 & 0.8792 & 28.91 & 0.8455 & 27.07 & 0.7962 & 25.63 & 0.7354 \\
\hline $\begin{array}{l}\text { ATFDF(Habib, Hussain, } \\
\text { et al., 2015) }\end{array}$ & 33.20 & 0.9602 & 32.72 & 0.9564 & 31.21 & 0.9236 & 29.93 & 0.9006 & 29.16 & 0.8887 \\
\hline FPDM(Turkmen, 2013) & 30.22 & 0.8810 & 29.16 & 0.8525 & 28.05 & 0.8273 & 27.29 & 0.7692 & 26.10 & 0.7172 \\
\hline MDW(Li et al., 2014) & 31.84 & 0.9176 & 30.09 & 0.8943 & 29.32 & 0.8533 & 27.99 & 0.7802 & 26.70 & 0.7201 \\
\hline NWM(L. Liu et al., 2015) & 32.39 & 0.9245 & 31.75 & 0.9007 & 29.22 & 0.8656 & 27.90 & 0.8028 & 26.98 & 0.7519 \\
\hline $\begin{array}{l}\text { LNI-MTR(Hussain } \\
\text { Dawood et al., 2017) }\end{array}$ & 35.70 & 0.9821 & 34.70 & 0.9604 & 33.62 & 0.9164 & 32.54 & 0.8425 & 31.45 & 0.7407 \\
\hline $\begin{array}{l}\text { DROAD(Hussain \& } \\
\text { Habib, 2017) }\end{array}$ & 34.15 & 0.9783 & 33.17 & 0.9672 & 31.89 & 0.9434 & 30.39 & 0.9222 & 29.68 & 0.9011 \\
\hline TOID(Azhar et al., 2018) & 36.51 & 0.9706 & 35.15 & 0.9646 & 34.11 & 0.9479 & 32.01 & 0.9323 & 31.86 & 0.9084 \\
\hline Proposed & 38.97 & 0.9810 & 37.43 & 0.9764 & 36.00 & 0.9685 & 34.77 & 0.9493 & 33.83 & 0.9288 \\
\hline
\end{tabular}

Table 5 Quantitative results of proposed image d-noising method on 10 images randomly selected from USC-SIPI database under various intensities of RVIN.

\begin{tabular}{|c|c|c|c|c|c|c|c|c|c|c|c|c|}
\hline \multirow{3}{*}{ Image } & \multicolumn{12}{|c|}{ RVIN intensity } \\
\hline & \multicolumn{4}{|c|}{$10 \%$} & \multicolumn{4}{|c|}{$30 \%$} & \multicolumn{4}{|c|}{$60 \%$} \\
\hline & PSNR & SSIM & MD & FD & PSNR & SSIM & MD & FD & PSNR & SSIM & MD & FD \\
\hline Couple & 42.10 & 0.9798 & 2155 & 2152 & 37.68 & 0.9378 & 5497 & 5537 & 34.34 & 0.8242 & 7970 & 8159 \\
\hline Elaine & 42.96 & 0.9872 & 1461 & 1547 & 38.36 & 0.9640 & 4244 & 4098 & 34.28 & 0.8589 & 5508 & 5556 \\
\hline Girl & 42.42 & 0.9564 & 694 & 614 & 38.64 & 0.9060 & 1829 & 1708 & 36.84 & 0.6917 & 1725 & 1669 \\
\hline JellyBeans & 43.88 & 0.9691 & 804 & 783 & 41.00 & 0.9328 & 2320 & 2261 & 38.83 & 0.8976 & 3340 & 3445 \\
\hline Girl & 45.50 & 0.9608 & 1035 & 1005 & 40.88 & 0.9209 & 2751 & 2778 & 36.75 & 0.8665 & 4598 & 4446 \\
\hline JellyBeans & 43.51 & 0.9659 & 789 & 833 & 40.05 & 0.9296 & 2301 & 2266 & 36.78 & 0.8704 & 3032 & 3129 \\
\hline Airplane & 43.12 & 0.9855 & 2868 & 3050 & 38.94 & 0.9606 & 7697 & 8299 & 34.00 & 0.8303 & 8191 & 8917 \\
\hline Girl & 44.22 & 0.9754 & 815 & 766 & 39.56 & 0.9265 & 2077 & 2057 & 34.90 & 0.8008 & 2968 & 2642 \\
\hline Tank & 41.68 & 0.9750 & 589 & 632 & 37.01 & 0.9249 & 1705 & 1751 & 34.05 & 0.7786 & 2853 & 2975 \\
\hline Splash & 45.82 & 0.9922 & 3201 & 3021 & 40.93 & 0.9760 & 8520 & 7822 & 36.59 & 0.8496 & 10872 & 10387 \\
\hline Average & 43.52 & 0.9747 & 1441 & 1440 & 39.31 & 0.9379 & 3894 & 3857 & 35.79 & 0.8369 & 5105 & 5132 \\
\hline
\end{tabular}

Along with the quantitative results, the superiority of the proposed denoising algorithm can also be observed visually in Figs (11-17) where the images taken from USC-SIPI database are used to compare the proposed method with state-of-the-art methods. The seven images selected from the database first 
converted into grayscale if found colored and then degraded with $50 \%$ intensity of RVIN. The images are of various sizes, resolutions and textures that indicate the denoising performance of the proposed method on a wide variety of images. It is clear from the denoising results shown in Fig. 11 that the proposed method gives better denoising results. The visual results of comparison methods show that they have small patches of noise. DROAD has very few amount of noise left but the visual result of proposed method show its improved denoising capability and better preservation of edges. Figure 12 also shows the better denoising and texture-preserving power of the proposed method as compared to other comparison filters shown. MSN and ATFDF have large amount of noise as shown while AFIDM is blurred to some extent. DROAD has better results but the edge preserving capability of the proposed method is better as compared to DROAD. It can be observed from the eyes and lip portion of the restored images which are restored in the proposed method, effectively.

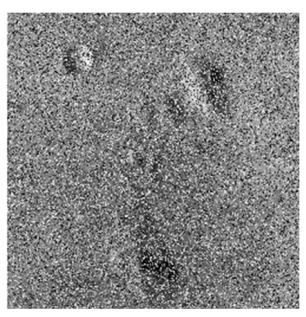

a) Noisy

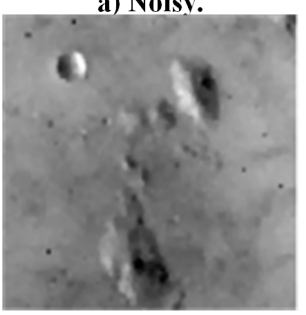

d) AFIDM.

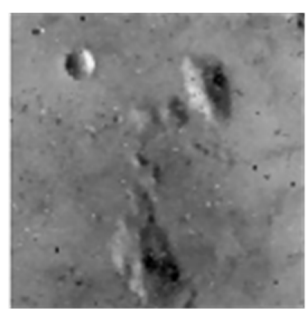

b) MSN.

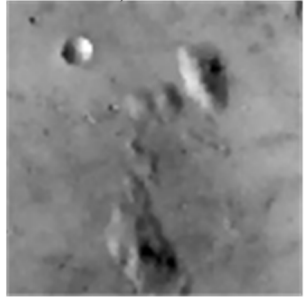

e) DROAD.

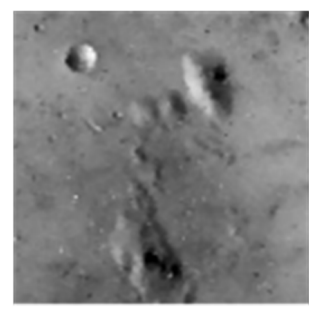

c) ATFDF.

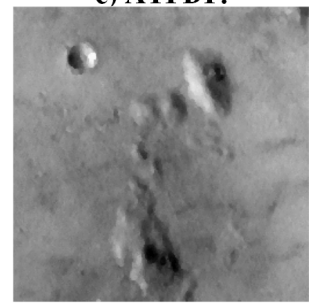

f) Proposed.

Fig.11 Visual comparison of proposed and state-of-the-art denoising methods on 'Moon surface' image taken from USC-SIPI database at $50 \%$ intensity of RVIN.

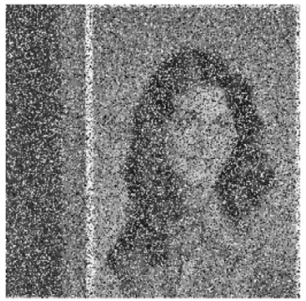

a) Noisy.

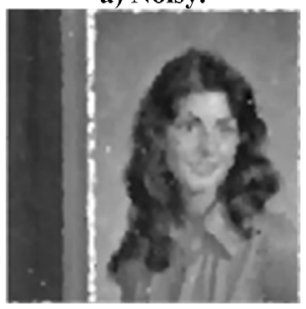

d) AFIDM.

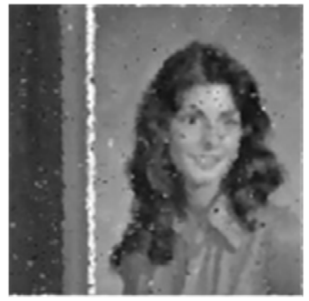

b) MSN.

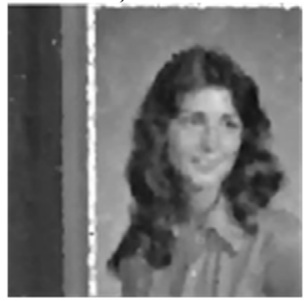

e) DROAD.

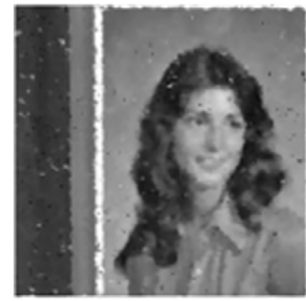

c) ATFDF.

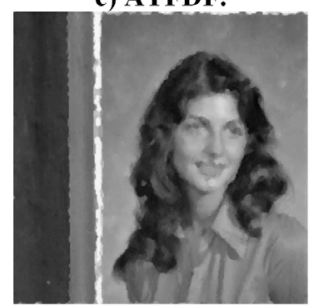

f) Proposed.

Fig.12 Visual comparison of proposed and state-of-the-art denoising methods on 'Female' image taken from USCSIPI database at $50 \%$ intensity of RVIN. 
The better denoising results of the proposed algorithm can also be seen in Figs 13-17. In all of them, MSN and ATFDF have noise pixels that are not restored by their respective noise filtering method. AFIDM has very few noisy pixels left to be recovered while DROAD has much better denoising power but less as compared to the proposed method. The proposed method preserves the edges and texture of images due to the extraction of textons that preserve the local texture information that leads to global texture preservation. Moreover, it identifies the corrupted pixels in an effective manner by using the local statistics of the textons that leads to better restoration of noisy pixels. The better denoising performance of the proposed algorithm is due to the ability of proposed noise filtering method to differentiate among noisy and noise-free pixels using fuzzy membership functions while restoring the intensity value of identified noisy pixels.

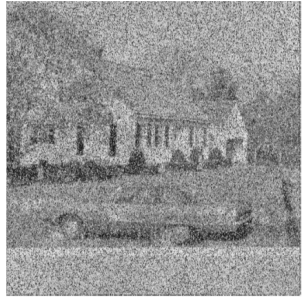

a) Noisy.

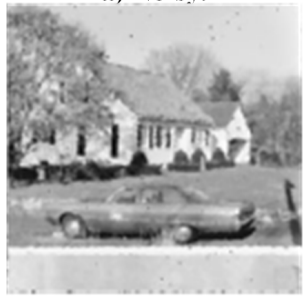

d) AFIDM.

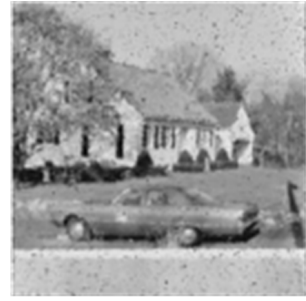

b) MSN.

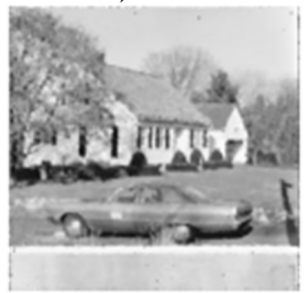

e) DROAD.

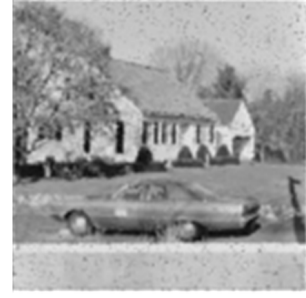

c) ATFDF.

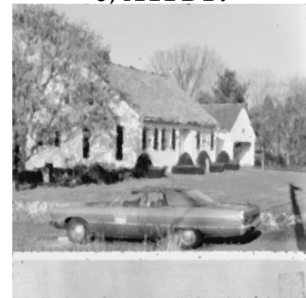

f) Proposed.

Fig.13 Visual comparison of proposed and state-of-the-art denoising methods on 'House' image taken from USCSIPI database at $50 \%$ intensity of RVIN.

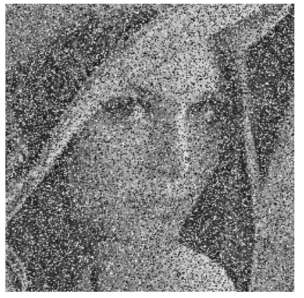

a) Noisy.

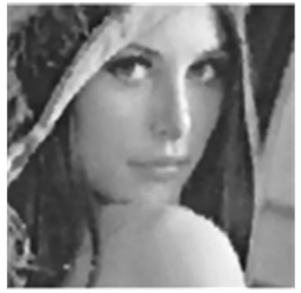

d) AFIDM

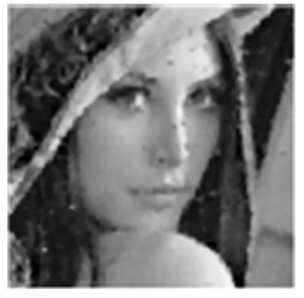

b) MSN.

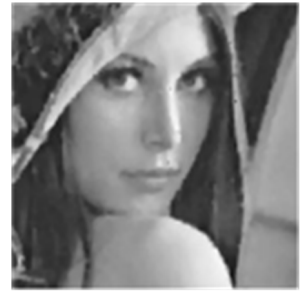

e) DROAD.

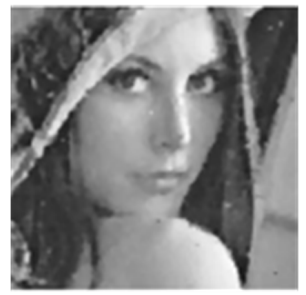

c) ATFDF.

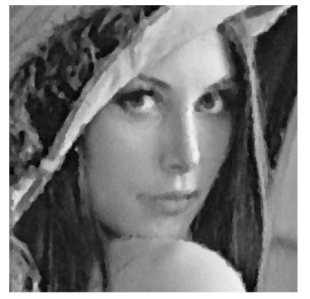

f) Proposed.

Fig.14 Visual comparison of proposed and state-of-the-art denoising methods on 'Lena' image taken from USCSIPI database at $50 \%$ intensity of RVIN. 


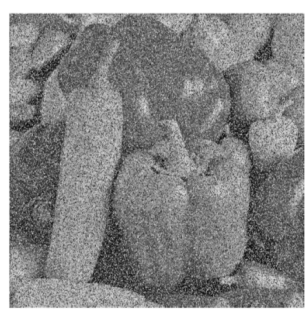

a) Noisy

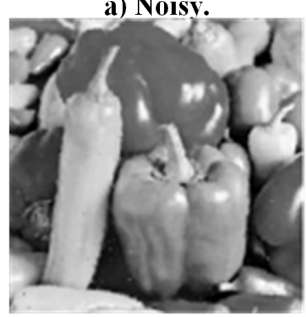

d) AFIDM.

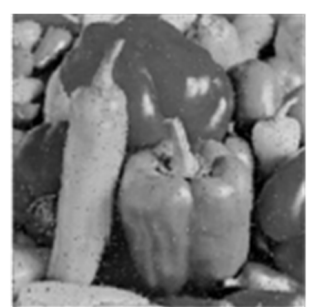

b) MSN.

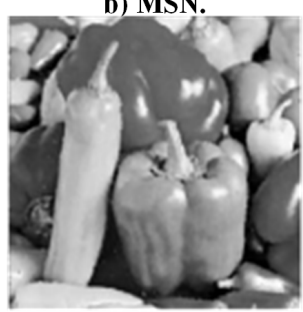

e) DROAD.

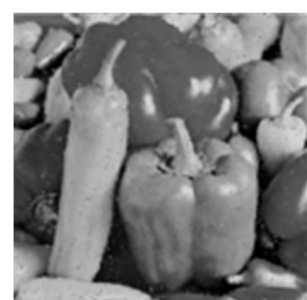

c) ATFDF

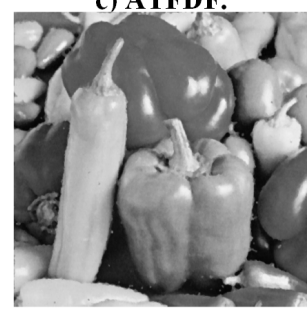

f) Proposed.

Fig.15 Visual comparison of proposed and state-of-the-art denoising methods on 'Peppers' image taken from USCSIPI database at $50 \%$ intensity of RVIN.

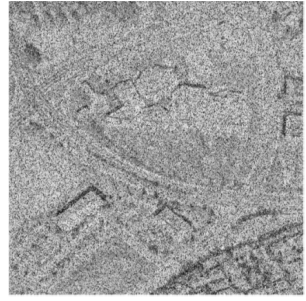

a) Noisy.

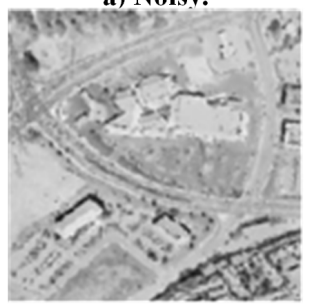

d) AFIDM.

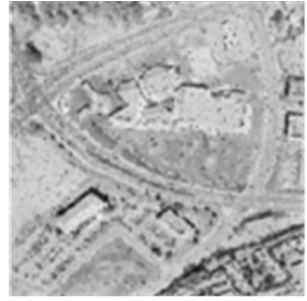

b) MSN.

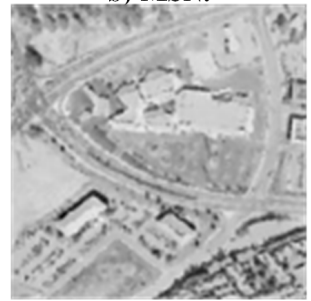

e) DROAD.

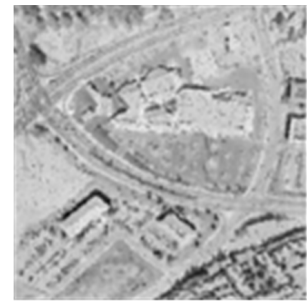

c) ATFDF.

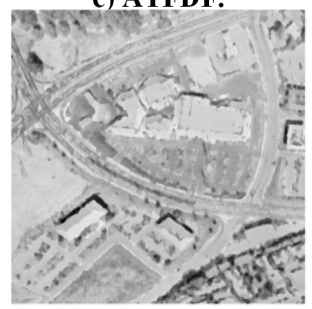

f) Proposed.

Fig.16 Visual comparison of proposed and state-of-the-art denoising methods on 'Aerial' image taken from USCSIPI database at $50 \%$ intensity of RVIN. 


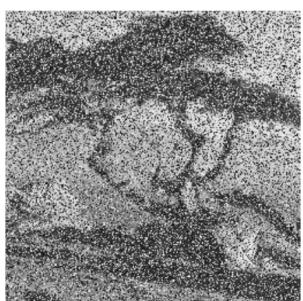

a) Noisv.

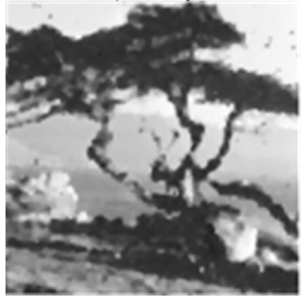

d) AFIDM.

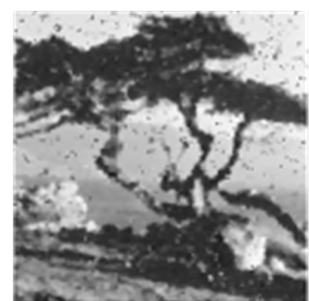

b) MSN.

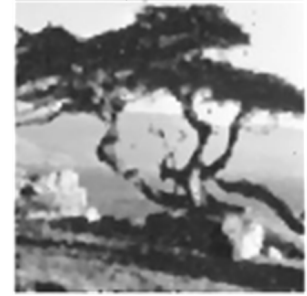

e) DROAD.

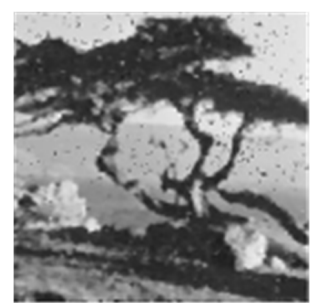

c) ATFDF.

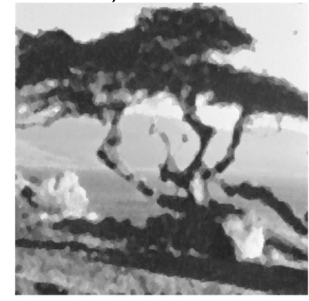

f) Proposed.

Fig.17 Visual comparison of proposed and state-of-the-art denoising methods on 'Tree' image taken from USC-SIPI database at $50 \%$ intensity of RVIN.

To evaluate the performance of the iterative nature of the proposed image denoising algorithm, the complete iterative reconstruction process on $60 \%$ RVIN corrupted Baboon image is shown in Fig. 18. Figure 18 (a) shows the original Baboon image while the image corrupted with $60 \%$ intensity of RVIN is shown in Fig. 18(b). The denoising results of the corrupted Baboon image are shown in Fig. 18 (c-i) after the execution of each iteration. It is clear from the images shown that as the number of iterations increases, so is the denoising power of the proposed algorithm. It is because with the increase in the number of executed iterations, more noisy pixels are identified and restored that are left to be identified in previous iterations. The visual results also show that the edge and detail-preserving power of the proposed algorithm also goes on increasing with increase in the number of iterations.

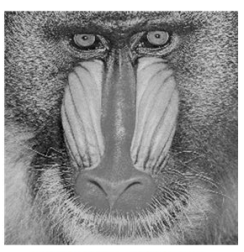

a) Original image.

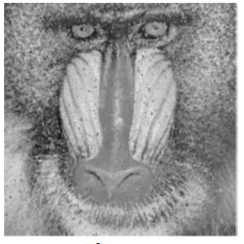

d) $2^{\text {nd }}$ iteration.

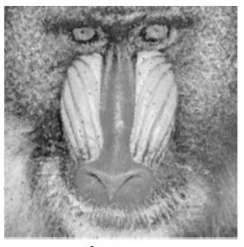

g) $5^{\text {th }}$ iteration.

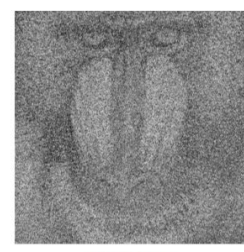

b) Noisy.

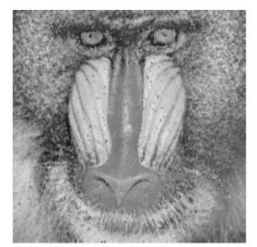

e) $3^{\text {rd }}$ iteration.

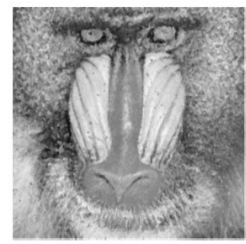

h) $6^{\text {th }}$ iteration.

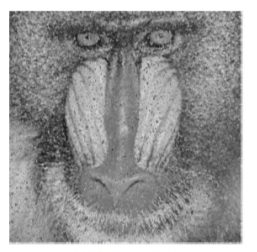

c) $1^{\text {st }}$ iteration.

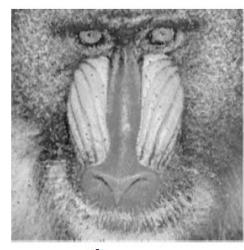

f) $4^{\text {th }}$ iteration.

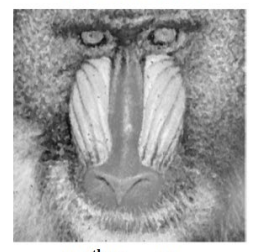

i) $7^{\text {th }}$ iteration.

Fig. 18 Visual results of proposed denoising method in an iterative manner on Baboon image corrupted with $60 \%$ intensity of RVIN. 
The robust denoising performance of the proposed algorithm can also be observed visually on biomedical images, as shown in Figs. 19-21. The restored biomedical images show the better restoration power of the proposed algorithm. The texture and edge preserving capability of the proposed algorithm for biomedical images can also be seen. The restored images ensure the proper diagnosis and treatment. It can be seen that the proposed denoising algorithm can restore biomedical images of various textures and resolution on different intensities of RVIN.

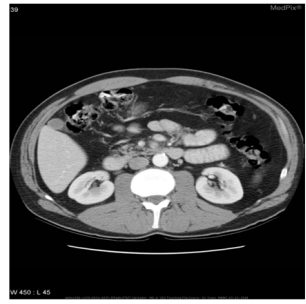

Original image.

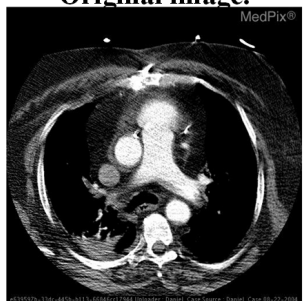

Original image.

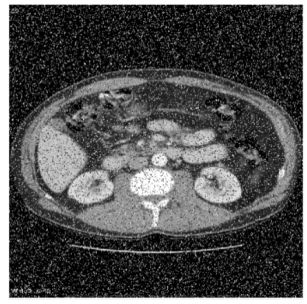

$20 \%$ noisy.

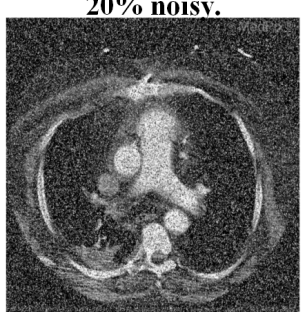

$40 \%$ noisy.

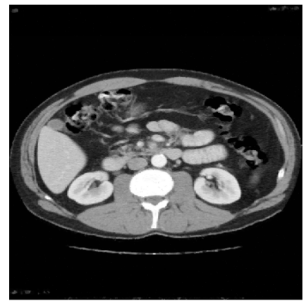

Restored image.

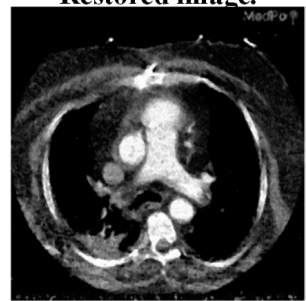

Restored image.

Fig. 19 Denoising results of proposed algorithm on CT-Scan images taken from MedPix database under various intensities of RVIN. First column shows original images of CT-Scan, Second and third columns show the images corrupted with RVIN and the restored images, respectively.

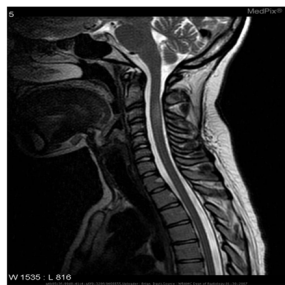

Original image.

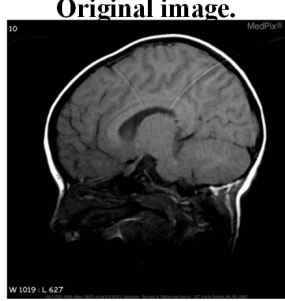

Original image.

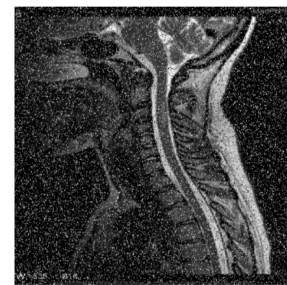

$20 \%$ noisy

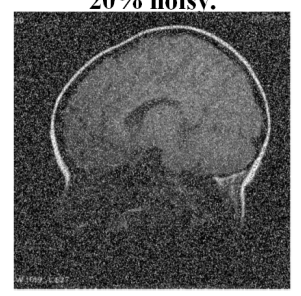

$40 \%$ noisy.

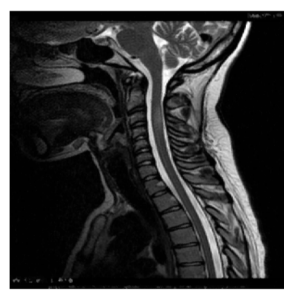

Restored image.

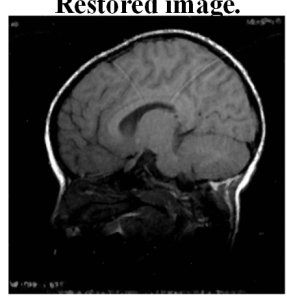

Restored image.

Fig. 20 Denoising results of proposed algorithm on MRI images taken from MedPix database under various intensities of RVIN. First column shows original images of MRI, Second and third columns show the images corrupted with RVIN and the restored images, respectively. 


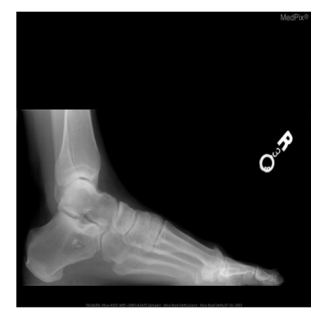

Original image.

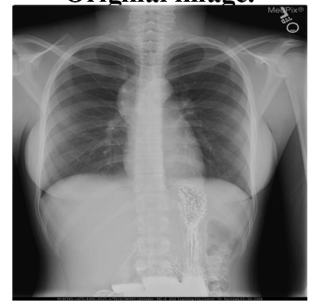

Original image.

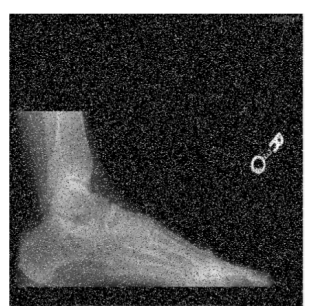

$20 \%$ noisv

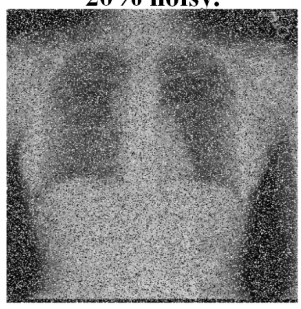

$40 \%$ noisy.

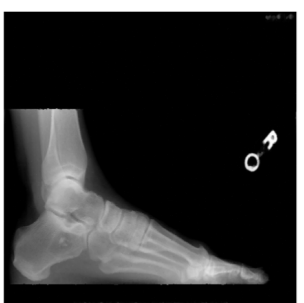

Restored image.

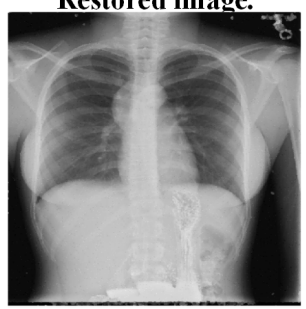

Restored image.

Fig. 21 Denoising results of proposed algorithm on X-Ray images taken from MedPix database under various intensities of RVIN. First column shows original images of X-ray, Second and third columns show the images corrupted with RVIN and the restored images, respectively.

\subsubsection{Parameters'setting}

A number of parameters that assists in the overall execution of the proposed image denoising algorithm are set according to the specific image used and the noise intensity. These parameters include the size of sliding window, the orientations of textons, the constant value used to define the threshold range and the maximum allowable iterations.

To decide on the size of sliding window, numerous experiments were conducted and a size of $5 \times 5$ is chosen for the proposed denoising algorithm. It is considered as the most suitable size as the distinction among noisy and noise-free pixels is difficult for smaller window sizes, especially for higher noise intensities. It is because for higher intensities of RVIN, half or more than half of the pixels of sliding window are corrupted and a noisy pixel is surrounded by many other noisy pixels hence, the identification becomes difficult among less number of pixels. A pre-defined threshold value is decided that is further used to estimate the threshold range to identify the corrupted pixels. We chose twenty-three as the best initial pre-defined constant value for lower intensities of RVIN while twenty-eight for the higher ones. The proposed denoising algorithm works in multiple iterations and the threshold range is varied in each iteration to identify more noisy pixels as compared to previous iterations. To vary the threshold range, we chose a constant value of three to decrement the threshold value in each iteration.

As textons are the fundamental elements of texture of images so, textons should be oriented in such a way that will preserve the texture and edge information of images, effectively. We propose textons by moving a $2 \times 2$ grid over the sliding window. As the distances among the pixels of a $2 \times 2$ grid is small, so the textons formed by it can better preserve the texture of images by keeping small distance. All pixels of the sliding window are properly involved in deciding the orientations of textons. Furthermore, the pixels are overlapping in two consecutive textons that preserve the salient edges lying in the direction of textons. The proposed algorithm works in an iterative manner to identify and restore majority of the noisy pixels. On the basis of great deal of experiments, we chose no more than five iterations for restoration of images corrupted with lower intensities of noise while, seven for the higher ones. Under such conditions, the proposed algorithm ensures better denoising results.

\subsubsection{Computational cost}

The computational cost is an important factor to evaluate an algorithm. The proposed denoising method extracts textons in various orientations of the sliding window. Furthermore, it works by executing 
multiple iterations to ensure better denoising performance. In both of them, more time is consumed that increases its computational cost. However, the high computational cost guarantees the better denoising and texture-preserving capability of the proposed algorithm.

\section{Conclusion}

To deal with the uncertainty encountered among noisy and edge pixels, a new iterative denoising algorithm is proposed for the restoration of gray-scale images corrupted with RVIN. Textons of various orientations are proposed to identify the RVIN corrupted pixels. The orientations of textons are decided by employing an isometric grid of minimum distance among its pixels that preserves the useful information of an image, effectively. The most similar tri-directional pixels obtained by applying fuzzy rules are used to estimate the intensity values of identified corrupted pixels. A comparison with various state-of-the-art denoising methods depicts the significant performance of the proposed algorithm both quantitatively and visually for low as well as higher intensities of RVIN. The main drawback of the proposed denoising algorithm is its high computational cost due to extraction of textons and execution of multiple iterations. For the future work, we will focus on the generation of textons in such orientations that will give restored images with better preservation of edges and texture. We will also try to apply texton-oriented denoising techniques on more complex noise models, for instance impulse-Gaussian mixed noise. Furthermore, the proposed algorithm can be modified in such a way to improve the denoising power for biomedical images. It can also be employed on a variety of biomedical images other than used in the paper, such as ECG, EEG, PET, SPECT and MEG etc.

\section{References}

Azhar, M., Dawood, H., \& Dawood, H. (2018). Texture-oriented image denoising technique for the removal of random-valued impulse noise. Journal of Electronic Imaging, 27(3), 33028.

Caiquan, J. L. X., \& Dehua, L. (2008). Adaptive center-weighted median filter. $J$ Huazhong Univ Sci Technol (Nat Sci Ed), 5-8.

Chen, T., \& Wu, H. R. (2001). Space variant median filters for the restoration of impulse noise corrupted images. IEEE Transactions on Circuits and Systems II: Analog and Digital Signal Processing, 48(8), 784-789.

Dawood, H., Dawood, H., \& Guo, P. (2015). Removal of random-valued impulse noise by local statistics. Multimedia Tools and Applications, 74(24), 11485-11498.

Dawood, H., Dawood, H., \& Guo, P. (2017). Generalization of Impulse Noise Removal. The International Arab Journal of Information Technology.

Dong, Y., \& Xu, S. (2007). A new directional weighted median filter for removal of randomvalued impulse noise. IEEE Signal Processing Letters, 14(3), 193-196.

Gonzalez, R. C. (2003). RE Woods Digital Image Processing using MATLAB. Prentice Hall,.

Gupta, V., Chaurasia, V., \& Shandilya, M. (2015). Random-valued impulse noise removal using adaptive dual threshold median filter. Journal of Visual Communication and Image Representation, 26, 296-304.

Habib, M., Hussain, A., \& Choi, T.-S. (2015). Adaptive threshold based fuzzy directional filter design using background information. Applied Soft Computing, 29, 471-478.

Habib, M., Hussain, A., Rasheed, S., \& Ali, M. (2016). Adaptive fuzzy inference system based directional median filter for impulse noise removal. AEU-International Journal of Electronics and Communications, 70(5), 689697.

Habib, M., Rasheed, S., Hussain, A., \& Ali, M. (2015). Random Value Impulse Noise Removal Based on Most Similar Neighbors. In Frontiers of Information Technology (FIT), 2015 13th International Conference on (pp. 329-333). IEEE.

Hussain, A., \& Habib, M. (2017). A new cluster based adaptive fuzzy switching median filter for impulse noise removal. Multimedia Tools and Applications, 76(21), 22001-22018.

Julesz, B. (1981). Textons, the elements of texture perception, and their interactions. Nature, 290(5802), 91-97.

Kang, C.-C., \& Wang, W.-J. (2009). Fuzzy reasoning-based directional median filter design. Signal Processing, 89(3), 344-351.

Kuykin, D., Khryashchev, V., \& Apalkov, I. (2009). Modified progressive switched median filter for image enhancement. In Proceedings of the International Conference on Computer Graphics and Vision (pp. 303-304).

Li, Z., Liu, G., Xu, Y., \& Cheng, Y. (2014). Modified directional weighted filter for removal of salt $\&$ 
pepper noise. Pattern Recognition Letters, 40, 113-120.

Liu, G.-H., Zhang, L., Hou, Y.-K., Li, Z.-Y., \& Yang, J.-Y. (2010). Image retrieval based on multitexton histogram. Pattern Recognition, 43(7), 2380-2389.

Liu, L., Chen, C. L. P., Zhou, Y., \& You, X. (2015). A new weighted mean filter with a two-phase detector for removing impulse noise. Information Sciences, 315, 1-16.

Lu, C.-T., Chen, M.-Y., Shen, J.-H., Wang, L.-L., Yen, N. Y., \& Liu, C.-H. (2018). X-ray bioimage denoising using directional-weightedmean filtering and block matching approach. Journal of Ambient Intelligence and Humanized Computing, 1-18.

Murugan, K., Arunachalam, V. P., \& Karthik, S. (2018). A hybird filtering approach for mri image with multiresolution. Cluster Computing, 1-6.

Rakshit, M., \& Das, S. (2018). An efficient ECG denoising methodology using empirical mode decomposition and adaptive switching mean filter. Biomedical Signal Processing and Control, 40, 140-148.

Sa, P. K., \& Majhi, B. (2010). An improved adaptive impulsive noise suppression scheme for digital images. AEU-International Journal of Electronics and Communications, 64(4), 322328.

Schulte, S., De Witte, V., Nachtegael, M., Van der Weken, D., \& Kerre, E. E. (2007). Fuzzy random impulse noise reduction method. Fuzzy Sets and Systems, 158(3), 270-283.

Schulte, S., Nachtegael, M., De Witte, V., Van der Weken, D., \& Kerre, E. E. (2006). A fuzzy impulse noise detection and reduction method. IEEE Transactions on Image Processing, 15(5), 1153-1162.

Toh, K. K. V., \& Isa, N. A. M. (2010). Cluster-based adaptive fuzzy switching median filter for universal impulse noise reduction. IEEE Transactions on Consumer Electronics, 56(4).

Turkmen, I. (2013). A new method to remove random-valued impulse noise in images. $A E U$ International Journal of Electronics and Communications, 67(9), 771-779.

Turkmen, I. (2016). The ANN based detector to remove random-valued impulse noise in images. Journal of Visual Communication and Image Representation, 34, 28-36.

Wang, Z., Bovik, A. C., Sheikh, H. R., \& Simoncelli, E. P. (2004). Image quality assessment: from error visibility to structural similarity. IEEE Transactions on Image Processing, 13(4), 600612.
Xiong, B., \& Yin, Z. (2012). A universal denoising framework with a new impulse detector and nonlocal means. IEEE Transactions on Image Processing, 21(4), 1663-1675.

Xu, Q., Li, Y., Guo, Y., Wu, S., \& Sbert, M. (2018). Random-valued impulse noise removal using adaptive ranked-ordered impulse detector. Journal of Electronic Imaging, 27(1), 13001. 